\title{
Positive und negative Koordination \\ in Verhandlungssystemen
}

Fritz W. Scharpf

93/1

Max-Planck-Institut für Gesellschaftsforschung

MPIFG Discussion Paper 93/1

Lothringer Str. 78

ISSN 0933-5668

D-5000 Köln 1

Februar 1993

Federal Republic of Germany

$\begin{array}{llll}\text { (RFC822): } & \text { mpi@mpi-fg-koeln.mpg.dbp.de } & \text { Telephone } & 0221 / 33605-0 \\ \text { (X400): } & \text { S=mpi;O=mpi-fg-koeln;P=mpg;A=dbp;C=de } & \text { Fax } & 0221 / 3360555 \\ \text { (PSI): } & \text { PSI\%4505011006::MPI } & & \end{array}$




\begin{abstract}
There is more coordination in the modern world than is plausibly explained by the classical mechanisms of community, market, hierarchy, and their commonly discussed variants. The paper explores modalities of non-market coordination whose appplication is not constrained by the narrow motivational and cognitive limitations of pure forms of hierarchical and negotiated coordination. The focus is on two varieties of negotiated self-coordinationhhh under conditions where actual negotiations are embedded in a preexisting structural context - either within hierarchical organizations or within selforganizing networks of cooperative relationships. Extrapolating from empirical findings in a variety of settings, it is argued that embeddedness will, at the same time, increase the scope of welfare maximizing "positive coordination" and create conditions under which externalities are inhibited through "negative coordination". In combination, these mechanisms are able to explain much of the de-facto coordination that seems to exist beyond the confines of efficient markets and hierarchies.
\end{abstract}

\title{
9310242
}

Max-Planck-Institut

fur Gesellschaftsforschung Bibliothek

PL $7-7 / 930 /$ In der modernen Welt gibt es mehr Koordination als durch die klassischen 25473000 Mechanismen der Gemeinschaft, des Marktes, der Hierarchie und ihrer üblicherweise erörterten Varianten erklärt werden könnte. Der Aufsatz diskutiert Modalitäten der nicht-marktlichen Koordination, deren Anwendung nicht durch die engen motivationalen und kognitiven Beschränkungen der reinen Formen hierarchischer und ausgehandelter Koordination begrenzt wird. Die Wirksamkeit dieser Modalitäten beruht auf der Einbettung von Verhandlungen in einen jeweils vorgegebenen strukturellen Rahmen - entweder einer hierarchischen Organisation oder eines selbstorganisierenden Netzwerks kooperativer Beziehungen. Erst diese Einbettung, so wird argumentiert, erweitert die Reichweite von wohlfahrtsmaximierender "positiver Koordination" und schafft Bedingungen, unter denen negative externe Effekte durch "negative Koordination" unterbunden werden können. Auch jenseits der Reichweite effizienter Märkte und Hierarchien können diese Mechanismen eine Erklärung für die faktische Wirksamkeit von Koordination liefern. 


\section{Inhalt}

Abstract

Einleitung

1. Das Koordinationsproblem

2. Grenzen der hierarchischen Koordination

Das Motivationsproblem

Das Informationsproblem

3. Grenzen der Verhandlungskoordination

Das Verhandlungsdilemma

$\begin{array}{lr}\text { Das Problem der großen Zahl } & 16\end{array}$

$\begin{array}{ll}\text { 4. Strukturell eingebettete Selbstkoordination } & 17\end{array}$

$\begin{array}{ll}4.1 \text { Selbstkoordination im Schatten der Hierarchie } & 18\end{array}$

Positive und negative Koordination 20

Die Bedeutung der Hierarchie $\quad 22$

4.2 Selbstkoordination in Netzwerken 23

$\begin{array}{ll}\text { Netzwerke als emergente Strukturen } & 24\end{array}$

Positive und negative Koordination in Netzwerken 31

5. Zusammenfassung 34

$\begin{array}{ll}\text { Literatur } & 35\end{array}$ 


\section{Einleitung}

Ein Charakteristikum der modernen Welt ist die zunehmend turbulente Interdependenz von Ereignissen und Entscheidungen. Ihre Antriebskräfte sind der wissenschaftlich-technische Fortschritt und der Zwang ökonomischer Konkurrenz. Ihre Begleiterscheinungen sind die immer raschere Innovation, die immer weiter differenzierte Arbeitsteilung und die immer vollständigere Mobilität von Kapital, Waren, Informationen und Personen. Zu ihren Folgeproblemen gehört die globale Umweltzerstörung ebenso wie die immer schnellere Entwertung vorhandener ökonomischer Kapazitäten, vorhandener Wissensbestände und kultureller Orientierungen und dementsprechend die Verarmung und Entwurzelung derer, die in den Turbulenzen der modernen Welt nicht mitschwimmen können.

Unter turbulenten Bedingungen wird die wechselseitige Abhängigkeit von Ereignissen und Handlungen nicht mehr durch stabile Ordnungen und kulturell gleichgerichtete Orientierungen unter Kontrolle gehalten. Die intentionale Koordination divergenter Erwartungen und kontingenter Pläne ist deshalb überlebenswichtig wie nie zuvor in der menschlichen Geschichte. Mit diesem Bedeutungszuwachs hat das wissenschaftliche Verständnis der Voraussetzungen und Wirkungsweisen von Koordination nicht Schritt gehalten. Die Ökonomie hat die theoretischen Grenzen der funktionsfähigen Marktkoordination so eng gezogen, daß der überwiegende Teil tatsächlichen Wirtschaftens auf dem weiten Feld des Marktversagens stattfindet; die Politikwissenschaft beschreibt eher die Erosion der hierarchischen Koordinationskapazität nationalstaatlicher oder hegemonialer Politik als die Effizienz supranationaler Steuerungskapazitäten; und die soziologische Theorie liefert plausiblere Argumente für die prinzipielle Unmöglichkeit der Kommunikation über Systemgrenzen hinweg als für die effektive Koordinationsleistung von Diskursen.

Im Vergleich zum gegenwärtigen Stand der Theorie scheint also weniger die allseits zunehmende Unordnung erklärungsbedürftig zu sein als das trotz allem real existierende $\mathrm{Ma} B$ an intra- wie interorganisatorischer, intra- wie intersektoraler und intra- wie internationaler Abstimmung und wechselseitiger Erwartungssicherheit. Offenbar gibt es jenseits der Grenzen von reinem Markt, hierarchischem Staat und herrschaftsfreien Diskursen in intern differenzierten und international verflochtenen modernen Gesellschaften mehr und wirksamere Koordinationsmechanismen, als die Wissenschaft bisher empirisch erfaßt und theoretisch begriffen hat. Mein Beitrag geht deshalb der Frage nach, wie das angesichts zunehmend turbulenter Verhältnisse 
immer noch erstaunlich hohe Maß tatsächlicher Handlungskoordination in modernen Gesellschaften theoretisch erklärt werden könnte.

\section{Das Koordinationsproblem}

Koordination wird hier als ein wohlfahrtstheoretisches Konzept verstanden. Anders gesagt, der Koordinationsbegriff soll hier Formen der Abstimmung bezeichnen, deren wohlfahrtstheoretisches Anspruchsniveau über das durch wechselseitige Antizipation in nicht-kooperativen Spielen erreichbare NashGleichgewicht hinausgeht. Zwar habe ich in einem früheren Aufsatz gezeigt, daß auch nicht-kooperative Spiele auf keineswegs selbstverständliche soziale Voraussetzungen der wechselseitigen Vorhersehbarkeit angewiesen bleiben. Aber koordiniertes Verhalten in dem hier gemeinten Sinn setzt darüber hinaus spezifische Einstellungen und institutionelle Mechanismen voraus, die inzwischen auch die Aufmerksamkeit institutionell orientierter Sozial- und Wirtschaftswissenschaftler finden (Lindberg et al. 1991).

Die theoretische Literatur hat sich vor allem auf die klassischen Mechanismen des Marktes und der Hierarchie (innerhalb von Organisationen oder im Staat) konzentriert. Darüberhinaus wird traditionellerweise auch "Gemeinschaft" (oder "Solidarität") noch zu den Koordinationsmechanismen gerechnet, und neuerdings haben auch "relationale Verträge" (Macneil 1978, Williamson 1985), "Clans" (Ouchi 1984), "Assoziationen" (Streeck/ Schmitter 1985) und "Netzwerke" wissenschaftliche Aufmerksamkeit gefunden. Analytisch scheinen einige dieser neueren Entdekkungen noch nicht eindeutig definiert. "Clans" könnte man als eine betriebswirtschaftliche Variante von "Gemeinschaft" verstehen und zugleich haben sie Ähnlichkeiten mit den "relationalen Verträgen"; andererseits erscheinen "Assoziationen" als schwache Variante einer hierarchischen Koordination, welche (wie der demokratische Verfassungsstaat) von der Zustimmung der Koordinierten abhängt. Schließlich könnte man auch "relationale Verträge" als charakteristische Form der Interaktion in Netzwerken interpretieren.

Es würde sich lohnen, diese Unklarheiten in einer systematischen Klassifikation auszuräumen. Dies kann hier ebensowenig geleistet werden wie ein vollständiger deskriptiver Überblick über die verfügbaren Koordinationsmechanismen. Im folgenden werde ich deshalb die Koordination durch traditionsgestützte (ethnische, sprachliche, religiöse oder auf Klassensolidarität basierende) "Gemeinschaften" ebenso vernachlässigen wie die (analytisch weitgehend geklärte) Marktkoordination. Stattdessen werde ich mich auf die 
Klärung der Voraussetzungen und Funktionsweisen bestimmter Formen der Nicht-Marktkoordination konzentrieren. Im Prinzip können diese auf Varianten der (demokratisch oder vertraglich legitimierten oder autoritären) hierarchischen Koordination und der (freiwilligen oder zwangsweisen) vertraglichen Koordination reduziert werden. Ich beginne zunächst mit einer Diskussion des Koordinationsproblems zwischen zwei Akteuren (Abb. 1).

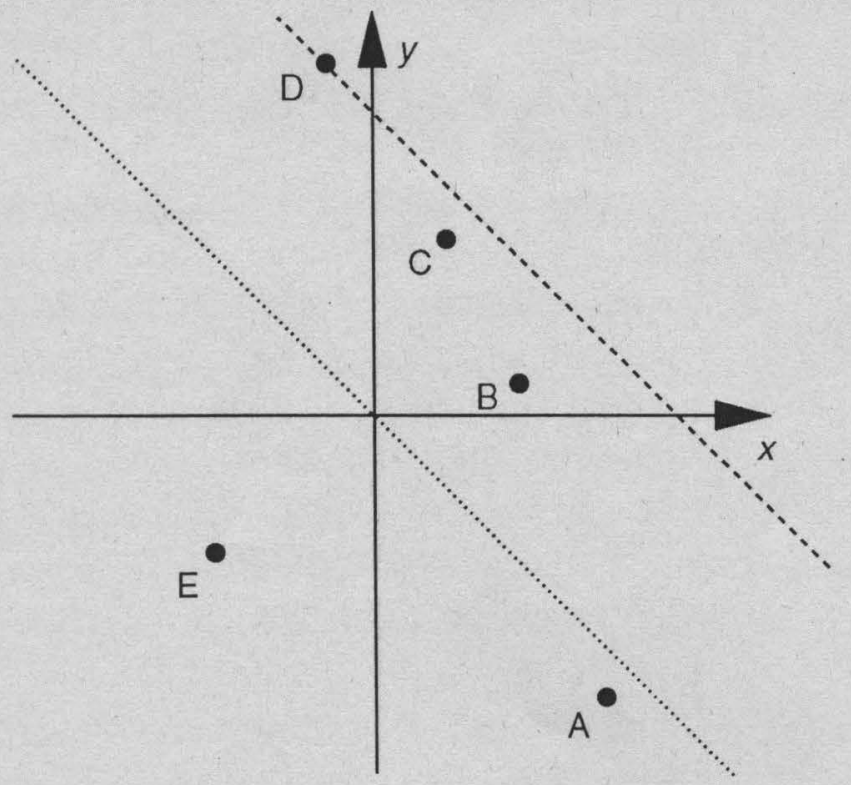

Abbildung 1: Das Koordinationssproblem

Vorausgesetzt werden zwei egoistisch-rationale Akteure ( $X$ und $Y$ ) mit voneinander unabhängigen (orthogonalen) Nutzenvektoren und eine Reihe von Handlungsoptionen (A), (B), (C), (D) und (E), die sich jeweils in ihrer Auswirkung für die Akteure unterscheiden. Der Status Quo wird durch den Ursprung des Koordinationsystems bezeichnet. Wenn man unterstellt, daß jeder der Akteure jede dieser Handlungsoptionen durch einseitige Entscheidung verwirklichen könnte, dann werden nur Vorhaben, die rechts von der Y-Achse liegen, für (X) akzeptabel sein und nur Vorhaben oberhalb der XAchse für $(Y)$. Dementsprechend würde $(X)$ die Projekte (D) und (E) ignorieren und er hätte eine Präferenz für Projekt (A), während (Y) die Projekte (A) und (E) ignorieren und das Projekt (D) präferieren würde.

Wenn man nun diese einseitig präferierten Lösungen wohlfahrtstheoretisch bewertet, dann wären unter dem Pareto-Kriterium beide nicht akzeptabel, weil jede von ihnen Verbesserungen gegenüber dem Status Quo für die eine Seite mit einer Verschlechterung für die andere Seite erkaufen würde. $\mathrm{Zu}$ einer differenzierenden Bewertung käme man dagegen bei Anwendung des 
(utilitaristischen) Kaldor-Kriteriums (Kaldor 1939). ${ }^{1}$ Der Unterschied wird. deutlich, wenn beide Projekte aus der Perspektive eines idealen hierarchischen Koordinators betrachtet werden - beispielsweise des Eigentümers eines Unternehmens mit zwei Abteilungen. Angesichts seiner Nutzenfunktion $\left(U_{C}\right.$ $=\mathrm{U}_{\mathrm{X}}+\mathrm{U}_{\mathrm{Y}}$ ) wäre offensichtlich kein Projekt unterhalb der Nordwest-SüdostDiagonalen überhaupt akzeptabel, und Vorhaben wären um so attraktiver, je weiter nordöstlich von der Diagonalen sie lokalisiert sind. Dementsprechend müßte der hierarchische Koordinator das von $(X)$ präferierte Projekt (A) verhindern; er würde jedoch das von (Y) präferierte Projekt (B) billigen, obwohl dadurch die Interessen von $(X)$ verletzt werden.

Was aber würde sich ändern, wenn die Koordination durch Verhandlungen zwischen $(X)$ und $(Y)$ statt durch hierarchische Entscheidung herbeigeführt werden müßte? Wenn wir von einem Zwangsverhandlungssystem ausgehen (so daß einseitige Aktionen ausgeschlossen sind), und wenn es ausschließlich um eindeutig fixierte Vorhaben geht, bei denen auch Ausgleichszahlungen nicht zugelassen werden, dann würde $(X)$ selbstverständlich alle Lösungen ablehnen, die links von der $Y$-Achse liegen, während $(Y)$ gegen alle unterhalb der X-Achse lokalisierten Projekte sein Veto einlegen müßte. Für eine Einigung kämen also überhaupt nur pareto-superiore Optionen innerhalb des nordöstlichen Quadranten in Betracht. Unter diesen würde freilich $(X)$ das Projekt (B) und $(Y)$ das Projekt $(C)$ präferieren, und für keinen von beiden gäbe es einsichtige Gründe dafür, warum gerade er den Wünschen des anderen nachgeben sollte. Der Versuch einer Koordination durch Verhandlungen stieße also auf zwei charakteristische Schwierigkeiten: Lösungen, welche die aggregierte Wohlfahrt (oder das "Gemeinwohl") maximieren, werden systematisch ignoriert, wenn sie nicht gleichzeitig auch für jeden einzelnen der Verhandlungspartner eine Verbesserung gegenüber dem Status Quo versprechen (d.h., wenn sie nicht innerhalb des nordöstlichen Quadranten der Abbildung lokalisiert sind); und wenn es mehr als eine pareto-superiore Lösung geben sollte, dann besteht immer auch die Gefahr, daß die Verhandlungen durch den Streit über die Wahl zwischen diesen Lösungen blokkiert werden. Daraus scheint $\mathrm{zu}$ folgen, daß das durch hierarchische Koordination erreichbare Wohlfahrtsniveau in der Regel höher liegt als das durch Verhandlungen erreichbare Ergebnis. Überdies kommt es, wenn die Zahl der Beteiligten zunimmt, zu einer Kumulation von Veto-Positionen,

1 Der utilitaristische Maßstab, der einen interpersonellen Nutzenvergleich voraussetzt, wird in der modernen Wohlfahrtsökonomie kritisch betrachtet (vgl. aber Selten 1986). Bei der Evaluierung von politischen oder organisatorischen Entscheidungen, welche die Wohlfahrt des Gemeinwesens oder den Gesamtgewinn eines Unternehmens maximieren sollen, kann man auf diesen Maßstab jedoch keineswegs verzichten (Hardin 1988; Hausman 1991). 
welche die Wahrscheinlichkeit rasch vermindert, daß überhaupt WohlfahrtsVerbesserungen durch multilaterale Verhandlungen zwischen egoistisch-rationalen Akteuren erreicht werden können. ${ }^{2}$

Freilich haben wir bisher unterstellt, daß es bei den Verhandlungen ausschließlich um eindeutig fixierte (also nicht kontinuierlich variable) Lösungen geht, und daß Ausgleichszahlungen, mit denen die Gewinner die Verlierer entschädigen könnten, nicht zugelassen sind. Wenn eine dieser Annahmen fallengelassen wird und wenn man überdies Transaktionskosten und Verteilungsprobleme vernachlässigt, dann folgt aus dem berühmten Coase-Theorem, daß alle Wohlfahrtsgewinne, die durch ideale hierarchische Koordination erreichbar wären, auch durch freiwillige Vereinbarungen zwischen autonomen und ausschließlich egoistisch-rationalen Akteuren realisiert werden können (Coase 1960). Bei freiwilligen Verhandlungen müßte die Partei, welche von einem Vorhaben Nachteile zu befürchten hätte, die daran Interessierten für den Verzicht bezahlen, ${ }^{3}$ während in einem Zwangsverhandlungssystem (etwa, wenn Eigentumsrechte gegen negative Externalitäten rechtlich geschützt sind) die Ausgleichzahlungen in die andere Richtung fließen müßten. In beiden Fällen -aber würde das Ergebnis von Verhandlungen zwischen egoistischrationalen Akteuren die aggregierte Wohlfahrt der Beteiligten maximieren. In Abbildung 1 beispielsweise hätte $(Y)$ durch die Verwirklichung des Vorhabens (A) mehr zu verlieren als $(X)$ dabei gewinnen würde; er könnte also in einem freiwilligen Verhandlungssystem dieses Projekt durch das Angebot von Ausgleichszahlungen verhindern. Umgekehrt hätte (Y) bei Projekt (D) mehr zu gewinnen als $(X)$ verlieren würde, und er könnte deshalb in einem Zwangsverhandlungssystem dessen potentielles Veto abkaufen. Unter beiden Bedingungen (und bei vernachlässigbaren Transaktionskosten) würden also nur Vorhaben oberhalb der Diagonalen überhaupt realisiert, und egoistischrationale Akteure würden sich überdies auf Lösungen einigen, die auf der am weitesten nordöstlich gelegenen Nutzen-Isoquante lokalisiert sind, und

2 Wie man aus Abbildung 1 entnehmen kann, wäre in einem Verhandlungssystem mit zwei Akteuren, orthogonalen Präferenz-Vektoren und zufallsverteilten Optionen die Wahrscheinlichkeit, daß die für einen Akteur attraktive Option auch für den anderen wenigstens akzeptabel wäre, $p=1 / 2$. Bei drei Akteuren fiele diese Wahrscheinlichkeit auf $1 / 4$, bei vier auf $1 / 8$, und bei $(N)$ Akteuren wäre die Wahrscheinlichkeit der Einigung nur noch $\mathrm{p}=1 / 2^{\mathrm{N}-1}$.

3 Allerdings ist die Gültigkeit des Coase-Theorems für den N-Personen-Fall und freiwillige Verhandlungen, die nicht durch den Schutz von Eigentumsrechten beschränkt werden (bei denen also auch partielle Koalitionen zum Nachteil Dritter zulässig sind), nach wie vor umstritten (Aivazian/ Callen 1981; Coase 1981; Aivazian et al. 1987). 
die demgemäß die aggregierte Wohlfahrt der Beteiligten (also deren "Gemẹinwohl") $)^{4}$ maximieren würden.

Nun beruhen freilich die Schlußfolgerungen des Coase-Theorems auf der Verfügbarkeit kontinuierlich variabler Lösungen oder der Möglichkeit von Ausgleichszahlungen. Beides sind plausible Annahmen unter Marktbedingungen oder jedenfalls in ökonomischen Kontexten, in dem Geld als gemeinsamer Maßstab für alle Arten von Werten fungiert. Dementsprechend können Ausgleichzahlungen oder Schattenpreise in der Tat die Koordination zwischen Unternehmen oder zwischen semi-autonomen Einheiten innerhalb von Unternehmen ermöglichen, und sie mögen auch für manche Arten von Verhandlungen unter öffentlichen Einrichtungen oder zwischen staatlichen Stellen und Akteuren im Privatsektor als adäquater Ausgleich akzeptabel sein. In vielen politischen Konstellationen lassen sich die relevanten Bewertungen jedoch nicht in Geldwerte übersetzen, oder Ausgleichszahlungen wären unter normativen Gesichtspunkten nicht akzeptabel (Baldwin 1990; Scharpf 1992; Zintl 1992). Aber auch dann wäre eine Annäherung an die Wohlfahrtseffekte des Coase-Theorems noch möglich, wenn separate Projekte mit komplementären Kosten-Nutzen-Bilanzen zu komplexen Verhardlungspaketen kombiniert werden können, durch die eine im ganzen ausgeglichene Interessenbilanz erreicht wird (Scharpf 1992). Dementsprechend wird sowohl in den internationalen Beziehungen wie in der Innenpolitik die Bedeutung von "issue-linkage" und "log-rolling" als Strategie zur Überwindung von Verhandlungsblockaden seit langem anerkannt (Haas 1980; Stein 1980; Sebenius 1983; MacGinnis 1986; Weingast 1989).

Aber auch wenn Hierarchie und Verhandlungen unter den postulierten idealen Bedingungen genau das gleiche Wohlfahrtsmaximum erreichen können, so folgt daraus noch lange nicht, daß beide Mechanismen auch unter realen Bedingungen wechselseitig substituierbar wären. In beiden Fällen kann das Optimum nur unter idealisierten Annahmen erreicht werden, für die praktikable Approximationslösungen allenfalls unter besonderen Bedingungen erreichbar erscheinen. Deshalb müssen zunächst einmal die praktischen Voraussetzungen erörtert werden, unter denen eine Annäherung an das Wohlfahrtsoptimum einerseits für die hierarchische Koordination und andererseits für die ausgehandelte Koordination erwartet werden könnte.

4 Sobald die Kompensation der Verlierer in Betracht gezogen wird, verliert die utilitaristische Definition des Gemeinwohls ihre autoritären Schrecken und könnte, so denke ich, von der normativen politischen Theorie mit erheblichem analytischem Gewinn eingesetzt werden. 


\section{Grenzen der hierarchischen Koordination}

Die Transaktionskosten-Ökonomie, so wurde jüngst behauptet, liefert zwar viele theoretische Gründe für das Versagen der Markt-Koordination, aber kaum Gründe gegen die Zusammenfassung aller ökonomischen Aktivitäten unter einheitlicher hierarchischer Leitung in einem einzigen riesigen Unternehmen (Milgrom/ Roberts 1990: 78). Dies erscheint vor allem deshalb bemerkenswert, weil ein anderer Zweig der neuen institutionellen Ökonomie, die Public-Choice-Theorie, jede Rechtfertigung für die Ausübung hierarchischer Autorität im öffentlichen Sektor in Frage zu stellen scheint. Aber obwohl sich die strukturellen Bedingungen unterscheiden, unter denen hierarchische Autorität im Unternehmen und im Staat ausgeübt wird, hängt doch die Effizienz der hierarchischen Koordination in beiden Sektoren von genau den gleichen funktionalen Voraussetzungen ab (Miller 1992) ${ }^{5}$. Auf höchster Abstraktionsebene formuliert setzt hierarchische Koordination sowohl die Lösung eines Motivationsproblems als auch die Lösung eines Informationsproblems voraus. Hierarchische Autorität ist nur dann normativ akzeptabel, wenn sie im Gesamtinteresse des Unternehmens oder des politischen Gemeinwesens (und nicht im privaten Interesse der jeweiligen Positionsinhaber) ausgeübt wird. Zugleich müssen die hierarchischen Koordinatoren, da realer Nutzen nur von den Bürgern oder den Organisationsmitgliedern an der Basis produziert werden kann, sich auf valide Information über die lokalen Bedingungen auf den unteren Ebenen der Hierarchie stützen und verlassen können. Beide Voraussetzungen sind grundsätzlich problematisch.

\section{Das Motivationsproblem}

Die erste Voraussetzung ist zum zentralen Thema der positiven PublicChoice-Theorie geworden. In ihren pessimistischen Varianten unterstellt sie auf allen Seiten nicht nur Egoismus, sondern amoralischen "Opportunismus"

5 Das Desinteresse der Transaktionskosten-Ökonomie für die Effizienzprobleme der hierarchischen Koordination läßt sich vermutlich auf die konventionelle Gleichsetzung des Unternehmensinteresses mit dem Eigeninteresse der Inhaber oberster Leitungspositionen (die als "residual claimants" modelliert werden Alchian/ Demsetz 1972) zurückführen. Wo diese Gleichsetzung in Frage gestellt wird, verweist man üblicherweise auf die externen Restriktionen des Marktes und auf die Exit-Optionen, die nicht nur Kunden und Zulieferern, sondern auch den Beschäftigten und Investoren des Unternehmens offen stehen. Demnach würde also ironischerweise die Effizienz der hierarchischen Koordination gerade dann zweifelhaft, wenn Marktversagen den Wettbewerb auf den Faktor- und Produktmärkten des Unternehmens beeinträchtigt. Nach derselben Logik müßte überdies die politische Konkurrenz auch ausreichen, um die Effizienz der Hierarchie im öffentlichen Sektor zu sichern. 
("self-interest seeking with guile": Williamson/ Ouchi 1981: 351); und dessen. Wirkung wird im politischen Prozeß noch verschärft durch allfällige Informationsasymmetrien und Kollektivhandelns-Probleme der Betroffenen. Deswegen, so wird gefolgert, sind Ausbeutung und Unterdrückung selbst in Demokratien charakteristisch für den öffentlichen Sektor. Wählermehrheiten werden die staatliche Macht gegen Minderheiten einsetzen; Interessentengruppen werden sich auf Kosten der Steuerzahler und der Verbraucher bereichern; politische Parteien und Parlamentsfraktionen werden die Tagesordnung der Politik und die Abstimmungsverfahren manipulieren; Bürokraten werden ihre Budgets und die Privilegien ihres Amtes maximieren; und Regierungen verfolgen ihren Machterhalt auf Kosten des Gemeinwohls. Unter solchen Annahmen können auch demokratische Verfahren die Steigerung der Wohlfahrt nicht gewährleisten; allenfalls können sie die chaotische Instabilität von Herrschaft sichern und dadurch vielleicht die Chancen nachhaltiger Ausbeutung (Riker 1982) vermindern.

Aber die Public-Choice-Theorie ist in sich widersprüchlich (Dryzek 1992). Wenn Opportunismus und Informationsasymmetrien generell unterstellt werden müßten, dann wären auch die von der normativen Variante dieser Theorie postulierten verfassungsrechtlichen Sicherungen (Brennan/ Buchanan 1985) völlig unwirksam. Schon aus Gründen der internen Konsistenz ist darum die normative politische Theorie und auch jede andere institutionalistische Theorie zu der Annahme gezwungen, daß menschliches Verhalten im Prinzip - wenn auch unvollkommen - an Normen orientiert sei. So beruht ja die Möglichkeit von Markttransaktionen im Privatsektor nicht nur auf der rechtlichen Durchsetzbarkeit von Eigentumsrechten und vertraglichen Ansprüchen, sondern auch auf sozialen Normen, welche die "normale" (aber eben gerade nicht die "opportunistische") Verfolgung von Eigeninteressen in Marktsituationen legitimieren. Im Gegensatz dazu gelten für das Verhalten in Organisationen (und zwar auch in Wirtschaftsunternehmen) ganz andere Normen, welche die direkte Verfolgung privater Eigeninteressen bei der Wahrnehmung von Organisationsrollen eng beschränken und die Organisationsmitglieder dazu anhalten, innerhalb der "zone of indifference" ihres Beschäftigungsvertrages (Simon 1951/ 1957; 1991) ihre Anstrengungen zur Förderung der Organisationsinteressen einzusetzen. Erst recht steht im demokratischen Verfassungsstaat die Ausübung öffentlicher Ämter unter der Norm der "Fremdnützigkeit" (Mirbach 1992). Regierungsmitglieder und Beamte sind durch ihren Amtseid auf die Verfolgung des Gemeinwohls verpflichtet; Parlamentsmitglieder sind gehalten, das wohlverstandene Interesse ihrer Wähler dem eigenen Interesse voranzustellen; und die gleichen Normen gelten im Verhältnis zwischen Funktionsträgern und Mitgliedern in politischen Parteien oder Interes- 
sengruppen. Ähnliche Ansprüche folgen aus Normen der professionellen Ethik in den freien Berufen, in der Wissenschaft oder in den Medien.

Theorien, welche diese normative "logic of appropriateness" (March/ Olsen 1989) völlig ignorieren sind also sowohl in ihrer empirischen Erklärungskraft ${ }^{6}$ als auch in ihrer präskriptiven Plausibilität defizitär. Aber auch wenn Normen von Bedeutung sind, so folgt daraus keineswegs die praktische Irrelevanz von Eigeninteressen. Die Möglichkeiten des Mißbrauchs hierarchischer Autorität bleiben eine immer gegenwärtige Gefahr. Wir haben deshalb allen Grund, nach institutionellen Strukturen zu suchen, deren Anreize eine größere Übereinstimmung zwischen persönlichen oder organisatorischen Eigeninteressen und normativen Verpflichtungen bewirken können. Dies ist die Logik des demokratischen Verfassungsstaates mit seiner Institutionalisierung der "checks and balances", des politischen Wettbewerbs und der Freiheiten der Meinungsäußerung, der Versammlung und der Vereinigung. Trotz vieler Parallelen bleibt aber der Unterschied zwischen der Public-ChoiceTheorie und der theoretischen Tradition des demokratischen Verfassungsstaates von kritischer Bedeutung: Institutionen, welche die vorhandenen normativen Orientierungen von Akteuren stärken und deren eigene Festlegung auf öffentliche Zwecke gegen allfällige Versuchungen schützen sollen, sind eine Sache; eine ganz andere Sache wäre der Versuch, mit den Mitteln einer geschriebenen Verfassung unüberwïndbare Sicherungen gegen Amtsinhaber zu schaffen, denen man eine ausschließliche Orientierung an den eigenen Privatinteressen und entschlossenen Opportunismus unterstellt. Die Geschichte vieler demokratischer Verfassungsstaaten hat gezeigt, daß es für das erste Problem praktikable Lösungen gibt. Im Gegensatz dazu wären institutionelle Vorkehrungen gegen das zweite Problem nicht nur nach den eigenen theoretischen Prämissen zum Scheitern verurteilt, sondern sie müßten gerade im Erfolgsfall jene Steuerungskapazität staatlicher Institutionen zerstören, auf welche moderne Gesellschaften in einer turbulenten Umwelt mehr denn je angewiesen sind.

6 Der empirische Einwand wird von Brennan und Buchanan (1985: 51-53) konzediert. Sie verteidigen deshalb die Unterstellung opportunistischen Verhaltens in erster Linie als "skeptische Fiktion" (Schuessler 1988) zur Anleitung risikoaverser Verfassungsgeber (1985: 55-59), - die aber selbst einer normativen Gemeinwohl-Orientierung bedürfen (1985: 146-150). Im Rahmen des "PrincipalAgent"-Ansatzes ist jedoch nachgewiesen worden, daß auch diese Rechnung nicht aufgehen kann: Es gibt keine Institution, die trotz opportunistischer Hierarchen Effizienz garantieren könnte (Bianco/ Bates 1990; Miller 1992). 


\section{Das Informationsproblem}

Trotzdem bleibt die hierarchische Koordination auch unter günstigen Umständen eine gefährliche Lösung. Zugleich wird weithin befürchtet, daß sie auch eine ineffiziente Lösung sein müsse. Für zentrale Entscheidungsinstanzen sei es schwierig oder sogar unmöglich, zutreffende Informationen über die lokalen Bedingungen von Problemen und Lösungsmöglichkeiten zu gewinnen; und selbst wenn diese Schwierigkeit zu überwinden wäre, könnte das kumulierte lokale Wissen in der Zentrale nicht effektiv verarbeitet und genutzt werden. Kurz, das wahrscheinliche Ergebnis von Zentralisierung wäre entweder extreme Informationsknappheit oder Informationsüberlastung der Zentrale - mit der Folge schlecht informierter und nicht problemgerechter Entscheidungen oder unendlicher Verzögerungen (Hayek 1945).

In der Transaktionskosten-Theorie wird diese Hayeksche Fundamental-Kritik an der Möglichkeit hierarchischer Koordination mit dem Verweis auf das Prinzip der "selektiven Intervention" beantwortet, demzufolge Vorgesetzte ihre Weisungen strikt auf Angelegenheiten beschränken sollten, die auf ihrer eigenen Organisationsebene entschieden werden müssen, während alles übrige Akteuren auf den unteren Ebenen mit ihrem vermutlich besseren Zugang zu lokaler Information zu überlassen sei (Williamson 1985: 133-135; Milgrom/ Roberts 1990). Dabei wird jedoch regelmäßig die strukturelle Vorbedingung einer effektiven hierarchischen Koordination übersehen, die Herbert Simon (1962; 1973) als "näherungsweise Zerlegbarkeit" ("near decomposability") konzeptualisiert hat. Sie impliziert ein bestimmtes Muster von Interaktionsbeziehungen, bei dem Interaktionen zwischen Akteuren, die einem gemeinsamen Vorgesetzten unterstellt sind, wesentlich wichtiger sein und häufiger auftreten müssen als Interaktionen mit Mitgliedern anderer Einheiten. Eine solche "modulare" Ordnung herzustellen, ist die primäre Aufgabe der Organisationsentwicklung (Scharpf 1977).

Aber die Konstruktion modularer Organisationen wird immer schwieriger und schließlich unmöglich, wenn die Interaktionen zwischen den Aufgabenbereichen wichtiger, häufiger und variabler werden. Wo dies der Fall ist, da stehen hierarchische Organisationen vor der unattraktiven Wahl, entweder die hierarchische Kompetenzverteilung trotzdem aufrecht zu erhalten oder den zentralen Koordinationsanspruch zu reduzieren und sich stattdessen auf die horizontale Selbstkoordination zwischen den Untereinheiten zu verlassen. Im ersten Falle werden Aufgabeninterdependenzen zwischen Organisationseinheiten auf gleicher Ebene ignoriert und die Koordinationsprobleme werden bis zum ersten gemeinsamen Vorgesetzten nach oben verlagert. Im Ergebnis werden dabei die Vorteile der Dezentralisierung und der selektiven 
Interaktion preisgegeben, die Tagesordnung der höheren Leitungsebenen wird überlastet und deren Informationsverarbeitungsfähigkeit überfordert. Wo dies geschieht, da werden die Voraussetzungen einer effizienten hierarchischen Koordination systematisch verletzt und die Organisation leidet unter den wohlbekannten Übeln der Überzentralisierung. Im zweiten Falle dagegen, wenn die Untergebenen auf eigene Faust mit der zunehmenden Aufgabeninterdependenz zwischen den Einheiten fertig werden müssen, verliert die Organisation alle Vorteile der hierarchischen Koordination und sieht sich statt dessen mit den zwar andersartigen aber keineswegs weniger gefährlichen Problemen der horizontalen Selbstkoordination konfrontiert.

\section{Grenzen der Verhandlungskoordination}

Innerhalb und zwischen Organisationen wird Selbstkoordination typischerweise durch Verhandlungen erreicht. Deren motivationale Voraussetzungen sind im Vergleich zu denen der hierarchischen Koordination wesentlich weniger anspruchsvoll. Die Geltung des Coase-Theorems ist nicht auf moralisch integre, gemeinwohlorientierte Koordinatoren angewiesen; zur Erreichung des Wohlfahrtsoptimums bedarf es hier nur egoistisch-rationaler Parteien. Die Beteiligten haben jedoch mit zwei Problemen von vergleichbarer Schwierigkeit zu kämpfen, dem Verhandlungsdilemma und dem Problem der großen Zahl.

\section{Das Verhandlungsdilemma}

Aus wohlfahrtstheoretischer Sicht hängt der Erfolg von Verhandlungen davon $a b$, daß die Parteien eine gemeinsame Vorgehensweise definieren können, durch welche ihre aggregierten Nutzen maximiert werden, und daß sie sich überdies auf die Verteilung der dabei anfallenden Kosten und Erträge einigen können. Offensichtlich handelt es sich hierbei aber um Aufgaben ganz unterschiedlicher Art, die in der hier gewählten grafischen Darstellung (Abbildung 1) als Bewegungen in zwei zueinander senkrecht stehenden Dimensionen erscheinen. Einerseits haben die Parteien bei ihrer Suche nach besseren Lösungen ein gemeinsames Interesse daran, sich (in der Südwest-NordostDimension) auf eine Nutzen-Isoquante hinzubewegen, die dem Status Quo möglichst weit überlegen ist. In dieser Hinsicht befinden sie sich also in einem reinen Koordinationsspiel, in dem optimale Ergebnisse von subjektiven Eịnstellungen abhängen, die Kreativität, offene Kommunikation, und vertrauensvolle Zusammenarbeit begünstigen (Pruitt 1981; Groom 1991; Häusler/ 
Hohn/ Lütz 1993). Gleichzeitig werden aber eigennützige Verhandlungsteilnehmer sich (in der Nordwest-Südost-Dimension) über die Lokalisierung einer koordinierten Lösung auf jeder Nutzen-Isoquante streiten. Wenn die Auseinandersetzung um feststehende Lösungen geführt werden muß, kann sie die Einigung überhaupt verhindern. Wenn Ausgleichszahlungen möglich sind, kann zwar jede feststehende Lösung so transformiert werden, daß das Ergebnis in einem Abschnitt der Nutzen-Isoquante innerhalb des nordöstlichen Quadranten zu liegen kommt, aber auch dann werden die Parteien sich noch über die Lokalisierung auf diesem Abschnitt streiten, und dieser Streit hätte immer noch alle Eigenschaften eines Nullsummen-Konflikts.

Nun gibt es zwar eine Reihe von normativen Lösungen für die Regelung von Verteilungskonflikten in Verhandlungssituationen, (Nash 1950; Kalai/ Smorodinsky 1975; Osborne/ Rubinstein 1990), die bei vollständiger Information rationalen Akteuren akzeptabel erscheinen sollten. Aber dies ändert nichts an der Tatsache, daß für das praktische Verhandlungsverhalten unter unvollständiger Information die "kompetitiven" Orientierungen und verhandlungstaktischen Manöver, die den Erfolg im Verteilungsstreit begünstigen, psychologisch inkompatibel sind mit den "kooperativen" Einstellungen und Verhaltensweisen, die der kreativen Suche nach insgesamt besseren Lösungen dienlich wären. Schlimmer noch, Akteure, die gutwillig zu dieser Suche beitragen, laufen Gefahr, in der Verteilungsfrage übervorteilt zu werden. Hier liegt der Kern des "Verhandlungsdilemmas" (Lax/ Sebenius 1986), welches oft zu suboptimalen Verhandlungsergebnissen führt oder eine Einigung sogar in Situationen verhindert, in denen koordiniertes Vorgehen für alle Beteiligten höchst vorteilhaft sein könnte.

\section{Das Problem der großen Zahl}

Die Schwierigkeiten der ausgehandelten Koordination steigen mit der Zahl der selbständig Beteiligten und ihrer interdependenten Handlungsoptionen und dementsprechend mit der Zahl der Transaktionen, die gleichzeitig zustande kommen müssen. ${ }^{7}$ Während die Theorie der kooperativen Spiele eine

7 Hier ist die Unterscheidung zwischen "pooled interdependence" und "reciprocal interdependence" (Thompson 1967, 54-55) von kritischer Bedeutung. Im ersten Fall braucht die Koordination nicht über "Standardisierung" hinauszugehen. Zwar mag es auch dabei Konflikte über die Wahl zwischen konkurrierenden Standards geben, aber wenn ein Standard einmal akzeptiert ist, können die Parteien über ihre verbleibenden Optionen autonom entscheiden. Der Zahl der Akteure, die sich an einem solchen Standard orientieren können, sind deshalb auch keine intrinsischen Grenzen gesetzt. Die große Zahl wird also nur unter den Bedingungen reziproker Interdependenz zum Problem, wenn das Gesamter- 
ganze Reihe analytischer Lösungskonzepte für multilaterale Verhandlungsoder Koordinationsprobleme entwickelt hat (Gastel/ Paelinck 1992), sind die meisten dieser Lösungswege von einer mathematischen Komplexität, die für reale Anwendungsfälle selbst von Analytikern nicht bewältigt werden kann (Dinar et al. 1992). Im Vergleich dazu ist die Erweiterung der NashVerhandlungslösung von zwei Personen- auf den N-Personen-Fall analytisch vergleichsweise leichter $\mathrm{zu}$ handhaben. Freilich werden dabei Zwangsverhandlungen unterstellt, welche die Bildung von Partial-Koalitionen ausschlieBen. Aber selbst diese Lösung verlangt, wenn sie sich aus den Verhandlungsprozessen selbst ergeben soll (und nicht von einem externen Analytiker vorgegeben wird), immer noch $(\mathrm{N} \times(\mathrm{N}-1) / 2)$ paarweise Vereinbarungen, von denen jede die jeweiligen Auswirkungen auf alle anderen Beteiligten berücksichtigen muß (Harsanyi 1977: 196 - 203). Jenseits sehr enger Grenzen müssen deshalb multilaterale Verhandlungen an prohibitiven Anforderungen an die Informationsverarbeitungs- und Konfliktregelungskapazität scheitern. Anders gesagt: Das Problem der großen Zahl beschränkt die Zahl der Beteiligten, unter denen komplexe Aufgabeninterdependenz durch Verhandlungskoordination bewältigt werden kann.

\section{Strukturell eingebettete Selbstkoordination}

Die hierarchische Koordination scheint für das Problem der großen Zahl weit weniger anfällig zu sein, weil hier ja die Handlungsoptionen der Untergebenen von einem gemeinsamen Vorgesetzten zu einer insgesamt optimalen Lösung verarbeitet werden können. Wenn also die Kontrollspanne eines jeden Vorgesetzten auf ein praktikables Maß beschränkt wird, dann müßten MehrEbenen-Hierarchien das Handeln einer prinzipiell unbegrenzten Zahl von Akteuren koordinieren können. Die kritische Vorbedingung dieses komparativen Vorteils bleibt freilich die Möglichkeit einer modularen Organisationsstruktur. Daraus folgt umgekehrt, daß auch die hierarchische Koordination auf engere Schranken stößt in einer Welt, in der reziproke Interdependenzen sich ausweiten und verdichten, und in der deshalb Interaktionen über intra- und interorganisatorische, intra- und intersektoralen, intra- und internationalen Grenzen hinweg nicht nur an Häufigkeit, sondern auch an kurzfristiger Veränderlichkeit zugenommen haben.

gebnis von den Entscheidungen aller Beteiligten über ihre interdependenten Optionen abhängt. Wenn jeder von (N) Beteiligten zwischen (S) Optionen zu wählen hat, dann erfordert die Identifikation eines Gesamt-Optimums die Überprüfung von $\left(\mathrm{S}^{\mathrm{N}}\right)$ Gesamt-Lösungen oder von $\left[\mathrm{N} \times(\mathrm{N}-1) / 2 \times \mathrm{S}^{2}\right]$ Paaren von Optionen (Scharpf 1972). 
Wir befinden uns also offenbar in einer theoretischen Sackgasse. Sowohl die hierarchische Koordination als auch die Verhandlungskoordination sind anfällig für opportunistisches Verhalten, das im ersten Falle Autoritätsmißbrauch und Ausbeutung, im zweiten das Verhandlungsdilemma zur Folge hat. Sollten diese Motivationsprobleme irgendwie bewältigt werden, dann wäre die potentielle Reichweite der Selbstkoordination durch Verhandlungen begrenzt durch den explosiven Anstieg der Komplexität multilateraler Verhandlungen bei einer steigenden Zahl von Beteiligten. Hierarchische Koordination wäre zwar im Prinzip auch bei einer sehr großen Zahl von Beteiligten wirksam - aber nur, wenn der gesamte Aufgabenbestand tatsächlich in voneinander näherungsweise unabhängige Module aufgeteilt werden kann. Mit zunehmender Häufigkeit und Veränderbarkeit von Interdependenz-Beziehungen stoßen jedoch modulare Organisationsformen - und damit auch die hierarchische Koordination - ebenfalls an unübersteigbare Grenzen. Aber wenn das so ist, wie soll man dann die Tatsache erklären, daß auch in hochkomplexen Konstellationen immer noch ein erstaunlich hohes Maß an Koordination erreicht werden kann? Die Antwort, die ich auf den folgenden Seiten skizzieren will, basiert auf dem Begriff der "Einbettung" ("embeddedness"). Insbesondere möchte ich zeigen, daß die engen Schranken der Verhandlungskoordination wesentlich erweitert werden können, wenn solche Verhandlungen entweder in hierarchischen oder in netzwerkartigen Strukturen eingebettet sind.

\subsection{Selbstkoordination im Schatten der Hierarchie}

In unseren Untersuchungen der Entscheidungsprozesse in der Ministerialorganisation haben Renate Mayntz und ich vor zwanzig Jahren keine Belege dafür gefunden, daß hierarchische Koordination in dem hier definierten Sinne ${ }^{8}$ in der ministeriellen Praxis eine erhebliche Rolle spielte, jedenfalls soweit es um die Leitungsebene der Ministerien und des Kanzleramtes ging. Stattdessen fanden wir vertikale Interaktionsbeziehungen, die einem "Dialogmodell" entsprachen, in dem Politiker und politische Beamte sich in erster Linie auf Fragen der politischen Realisierbarkeit und Wünschbarkeit der diskutierten Lösungen konzentrierten, während Fragen der technischen Wirksamkeit und administrativen Praktikabilität in erster Linie von den Berufsbe-

8 Das soll selbstverständlich nicht heißen, daß auch in allen anderen Angelegenheiten - etwa Personalfragen, Organisationsfragen oder Einzelfallentscheidungen in der Sache - hierarchische Entscheidungen selten seien. Unsere These bezieht sich nur auf den Versuch der hierarchischen Koordination zwischen interdependenten Einzelprogrammen. 
amten in die Diskussion eingebracht wurden (Mayntz/ Scharpf 1975: 100107). Selbstverständlich hatten die Politiker gegenüber den Beamten das letzte Wort. Aber diese Möglichkeit spielte so gut wie keine Rolle, weil ja die Entscheidungsprämissen, die von den Beamten repräsentiert wurden, im Dialog nicht ignoriert werden konnten und auch nicht ignoriert wurden. Im Prozeß der Politikvorbereitung hatten Lösungsvorschläge überhaupt nur dann eine Chance, wenn sie nach beiden Kriterien mindestens akzeptabel waren (Simon 1964); was auch bedeutet, daß der Politikdialog in der Regel durch Einvernehmen und nicht durch hierarchischen Oktroi beendet wurde.

Wenn in der vertikalen Interaktionsbeziehung einvernehmliche Entscheidungen die Regel waren, so galt dies erst recht für die horizontalen Verhandlungen zwischen Referaten und Abteilungen innerhalb des gleichen Ministeriums oder zwischen den Ministerien. Faktisch sind ja alle wichtigen Programminitiativen, die innerhalb einer Organisationseinheit erwogen werden, funktional abhängig von Beiträgen anderer Einheiten, oder sie haben Auswirkungen auf den Zuständigkeitsbereich oder die Klientelgruppen anderer Einheiten, oder die Verwirklichung von übergreifenden Programmzielen ist von vornherein auf gemeinsame Anstrengungen mehrerer Einheiten angewiesen. Unter solchen Bedingungen ist Koordination offensichtlich wünschenswert und oft unerläßlich für eine erfolgreiche Regierungspolitik. Aber angesichts der "selektiven Perzeption" spezialisierter Organisationseinheiten (Dearborn/ Simon 1958) und ihrer Identifikation mit den Interessen ihrer jeweiligen Klientelgruppen, sind Programminitiativen innerhalb der Bürokratie in aller Regel umstritten. Nun könnte zwar bei solchen Konflikten in jedem einzelnen Fall eine koordinierte Lösung von dem nächsten gemeinsamen Vorgesetzten und schließlich vom Kabinett oktroyiert werden; aber der Versuch, alle interministeriellen Konflikte "streitig ins Kabinett" zu bringen, wäre in jedem Fall zum Scheitern verurteilt - und das gleiche gilt für die Leitungsebene im Ressort. Mit anderen Worten: hierarchische Koordination ist zwar nicht ausgeschlossen, aber sie muß - wenn nicht Entscheidungsüberlastung zum Stillstand der Regierung führen soll - eine seltene Ausnahme bleiben. Die Minister und das Kabinett können ihre Funktionen überhaupt nur dann erfüllen, wenn die auf ihre Tagesordnung kommenden Entscheidungsvorschläge in aller Regel aus intra- und interministeriellen Abstimmungsprozessen hervorgehen, in denen bereits Einvernehmen zwischen den beteiligten Einheiten erreicht wurde. Das Überraschende ist, daß angesichts komplexer Interdependenzen und allfälliger Konflikte diese Übereinstimmung zwischen den Arbeitseinheiten in der Regel auch erreicht wird. 


\section{Positive und negative Koordination}

In unseren Untersuchungen haben wir das vorgefundene Lösungsmuster als Kombination zweier klar unterschiedener Formen der Selbstkoordination beschrieben, für die wir die Bezeichnungen "positive" und "negative" Koordination vorgeschlagen haben (Scharpf 1972). Die beiden Formen unterscheiden sich in ihrem inhaltlichen Anspruchsniveau und in ihren prozeduralen Anforderungen.

Inhaltlich kann man die positive Koordination als Versuch beschreiben, die Effektivität und Effizienz der Regierungspolitik insgesamt durch die Nutzung der gemeinsamen Handlungsoptionen mehrerer Abteilungen oder Ressorts zu steigern. Analytisch ist das Ziel der positiven Koordination also identisch mit der Maximierung aggregierter Wohlfahrtseffekte durch die idealisierten Modelle der hierarchischen Koordination und der Verhandlungskoordination. Im Gegensatz dazu erscheint das Anspruchsniveau der negativen Koordination begrenzter. Ihr Ziel ist die Vermeidung der Störungen, welche die ausschließlich an den eigenen Zielen orientierten Programminitiativen einer spezialisierten Einheit in den Zuständigkeitsbereichen anderer Einheiten auslösen könnten. Wohlfahrtstheoretisch formuliert sichert die erfolgreiche Negativkoordination also die Pareto-Superiorität neuer Politikinitiativen, während die positive Koordination das anspruchsvollere Kaldor-Optimum zu erreichen versucht.

Prozedural läuft positive Koordination fast immer auf multilaterale Verhandlungen in intra- oder interministeriellen Projektgruppen hinaus, deren Mandat die Berücksichtigung aller Handlungsoptionen aller beteiligten Einheiten einschließt. Im Gegensatz dazu erfolgt negative Koordination typischerweise durch bilaterale "Abstimmung" zwischen der an einer Programminitiative arbeitenden Einheit und anderen Einheiten, deren Zuständigkeitsbereich potentiell betroffen werden könnte - wobei deren eigene Handlungsoptionen in der Regel nicht zur Disposition stehen. Weil dies so ist, reduziert sich die Abstimmung oft auf eine bloße Formalität, wenn die initiierende Einheit die Einwände der "Mitzeichnungsberechtigten" antizipiert und bei der Formulierung ihres Programmvorschlags schon berücksichtigt hat.

Aus alledem folgt, daß die negative Koordination die Komplexität der horizontalen Selbstkoordination und damit die Bedeutung des Problems der großen Zahl drastisch reduziert (Abbildung 2). ${ }^{9}$ Ebenso klar ist allerdings,

9 Bei (N) Einheiten mit jeweils (S) Programm-Optionen erfordert die positive Koordination die gleichzeitige Überprüfung von $\left[\mathrm{N} \times(\mathrm{N}-1) / 2 \times \mathrm{S}^{2}\right]$ Beziehungen 


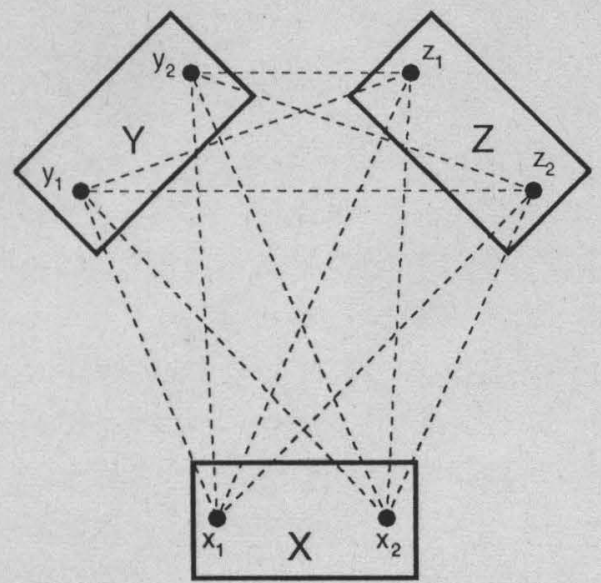

Positive Koordination

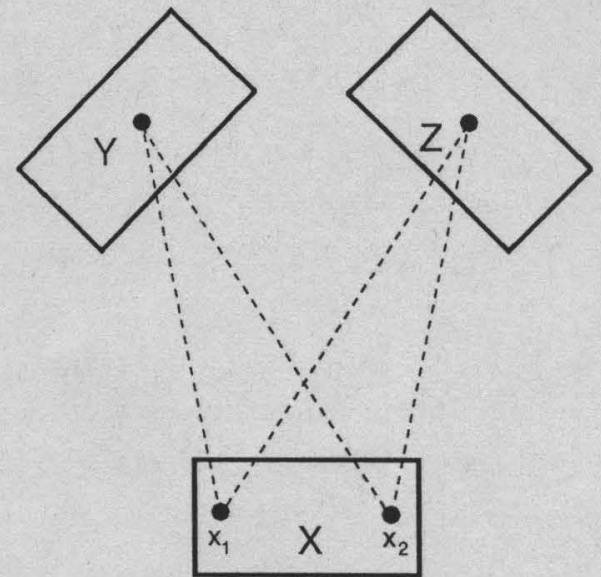

Negative Koordination

Abbildung 2: Positive und negative Koordination

daß der Spielraum für Programminnovationen vermindert wird, wenn nur die Handlungsoptionen der initiierenden Einheit zur Disposition stehen, während bei allen mitzeichnenden Einheiten der jeweilige Status Quo ihrer Programme als unveränderliche Gegebenheit vorausgesetzt wird. Bei einer steigenden Zahl von Beteiligten führt negative Koordination deshalb zu einer Kumulation von Veto-Positionen, welche die erreichbaren Wohlfahrtsgewinne unter das Niveau drückt, das durch positive Koordination erreicht werden könnte (Anmerkung 2 oben). Wenn man freilich die Ergebnisse der negativen Koordination mit den Wohlfahrtsverlusten vergleicht, die bei unkoordiniertem Vorgehen zu erwarten wären, so erscheint sie immer noch durchaus attraktiv; und wenn positive und negative Koordination kombiniert werden können, dann sind Annäherungen an das Wohlfahrtsoptimum möglich. In der Ministerialorganisation jedenfalls liegt hier die Erklärung dafür, wie trotz eines sehr hohen Grades komplexer Aufgaben-Interdependenz ein brauchbares Niveau effektiver Programmkoordination erreicht werden kann.

zwischen interdependenten Optionen. Die negative Koordination erfordert dagegen nur die Überprüfung von [S $\times(\mathrm{N}-1)$ ] Interaktionsbeziehungen, die überdies sequentiell abgearbeitet werden können. 


\section{Die Bedeutung der Hierarchie}

Technisch kann man die positive wie die negative Koordination als Formen der horizontalen Selbstkoordination (und nicht der hierarchischen Koordination) beschreiben. Aber eine solche Beschreibung ignoriert die fortdauernde Bedeutung hierarchischer Strukturen im ministeriellen Entscheidungsprozeß. Der Erfolg der positiven Koordination wird wesentlich begünstigt durch die Tatsache, daß die Verhandlungen zwischen den Untereinheiten typischerweise im Auftrag der Leitung stattfinden und daß ihre Ergebnisse entweder vom Minister oder vom Kabinett und vom Kanzler akzeptiert werden müssen. Deshalb ist es allen Beteiligten klar, daß der relative Erfolg oder Mißerfolg der Verhandlungen nach den übergreifenden Nutzenkriterien der höheren Ebene beurteilt werden. Überdies haben, da die Ergebnisse auf der höheren Ebene überprüft werden, auch unredliche Verhandlungstaktiken zwischen den Beteiligten weniger Aussicht auf Erfolg. Dementsprechend wird das "Verhandlungsdilemma", an dem Versuche der Verhandlungskoordination so oft scheitern, zumindest wesentlich gemildert, wenn die Verhandlungen innerhalb einer hierarchischen Organisationsstruktur stattfinden. Ebenso verdankt die Negativkoordination in der Ministerialbürokratie ihre Wirksamkeit zum großen Teil autoritativ vorgegebenen Verfahrensregeln und letzten Endes der Erwartung, daß das letzte Wort beim Minister oder beim Kabinett liegen wird, und daß diese einseitige Programminitiativen kaum ratifizieren werden, solange intra-ministerielle und interministerielle Programmkonflikte noch nicht ausgeräumt sind.

Hierarchische Strukturen, auch wenn sie eine wirksame hierarchische Koordination nicht leisten können, definieren also immerhin den Kontext, innerhalb dessen Verhandlungen stattfinden müssen. Einerseits schafft die hierarchische Organisation Bedingungen für Zwangsverhandlungen, durch die einseitige Aktionen aus dem Handlungsrepertoire der Akteure eliminiert werden; andererseits schafft sie Bedingungen, welche die Versuchungen beseitigen oder jedenfalls mindern, die andernfalls die Parteien in der Falle des "Verhandlungsdilemmas" festhalten würden.

Kurz: die effektive Koordinationsleistung von Verhandlungen kann wesentlich gesteigert werden, wenn diese in der hierarchischen Struktur der Ministerialbürokratie eingebettet sind. Und da hierarchische Organisationsstrukturen in der modernen Welt allgegenwärtig sind, kann man auch mit guten Gründen vermuten, daß hierarchisch eingebettete Verhandlungen weithin verfügbar sind, um Probleme der komplexen Aufgaben-Interdependenz innerhalb formaler Organisationen zu bewältigen. 
Überdies kann die Logik des Modells von Verhandlungen innerhalb hierarchischer Organisationen auf Beziehungen übertragen werden, die unter der hierarchischen Autorität des Staates stehen. In den meisten westlichen Demokratien wurde zwar in der Politik-Formulierung ebenso wie in der PolitikImplementierung die einseitige Ausübung staatlicher Autorität weithin verdrängt durch formelle oder informelle Verhandlungen zwischen staatlichen Akteuren und den betroffenen Individuen oder Organisationen. Zugleich werden wichtige Bereiche von öffentlichem Interesse durch Verhandlungen innerhalb pluralistischer oder korporatistischer "Politiknetzwerke" gestaltet (Scharpf 1991). Während das erste Muster dem vertikalen "Dialogmodell" entspricht, das oben beschrieben wurde, entspricht das zweite der horizontalen Selbstkoordination. Aber in beiden Dimensionen geht es hier typischerweise um Verhandlungen im Schatten hierarchischer Autorität. In vielen Bereichen werden die ausgehandelten Kompromisse auf höherer Ebene überprüft, und oft müssen die Vereinbarungen zwischen den organisierten Interessen letztlich durch administrative oder legislative Entscheidung in bindendes Recht umgesetzt werden. Ebenso wie in der Ministerialbürokratie impliziert hier die staatliche Zustimmung oder Ratifikation auch die Möglichkeit einer Ablehnung und damit die Fähigkeit, faire Verhandlungen zu erzwingen und offensichtlich opportunistische Strategien zu blockieren. Oft hat überdies der Staat die Gruppen und kooperativen Akteure bestimmt (oder sogar geschaffen), auf deren Zustimmung es bei den Verhandlungen ankommt; und ebenso werden die Verfahrensregeln, unter denen die Einigung erzielt werden muß, oft vom Staat bestimmt (Czada 1991; Lindberg/ Campbell 1991).

\subsection{Selbstkoordination in Netzwerken}

Zwar wird die Reichweite der Selbstkoordination erheblich erweitert, wenn Verhandlungen in hierarchische Autoritätsstrukturen eingebettet sind, aber damit wäre in allen jenen Fällen noch nichts gewonnen, wo der Koordinationsbedarf die Grenzen hierarchischer Zuständigkeiten überschreitet. Viele der kritischen Interdependenzen sind interorganisatorischer, intersektoraler oder zwischenstaatlicher Art, und wenn sie überhaupt bewältigt werden können, so sind sie auf die Möglichkeit einer "Koordination ohne Hierarchie" (Chisholm 1989) angewiesen. Im folgenden möchte ich zeigen, daß einige Funktionen, die ich der Existenz hierarchischer Strukturen zugeschrieben habe, auch von selbst-organisierenden Netzwerken vertrauensvoller Dauerbeziehungen erfüllt werden können. 


\section{Netzwerke als emergente Strukturen}

Ebenso wie der Begriff der Hierarchie wird auch der des Netzwerks zur Beschreibung bestimmter Interaktionsarten wie bestimmter Beziehungsstrukturen verwandt. Da es hier um ein funktionales Äquivalent für hierarchische Organisationsformen geht, interessiert vor allem die strukturelle Bedeutung. In der Literatur variieren zwar die Definitionen, aber die Betonung liegt im allgemeinen auf dem informellen (nicht-organisierten) und reziproken (nichthierarchischen $)^{10}$ Charakter von relativ dauerhaften Beziehungen zwischen mehr als zwei Akteuren. Unklar bleibt dabei, ob der Begriff auf Akteur-Konstellationen beschränkt bleiben soll, deren dyadische Beziehungen durch ein hohes Niveau gegenseitigen Vertrauens charakterisiert sind, oder ob das Vorhandensein oder Fehlen von Vertrauen als eine empirische Variable behandelt werden soll. In jedem Falle ist jedoch $\mathrm{zu}$ unterscheiden zwischen den (möglicherweise höchst unterschiedlichen) Eigenschaften der einzelnen Interaktionen und den Eigenschaften der dauerhaften Netzwerk-Beziehung, in der diese Interaktionen stattfinden.

Relativ dauerhafte Netzwerkstrukturen können auf Familien- oder Freundschaftsbindungen beruhen. Sie können aber auch durch Gesetz oder Verwaltungsakt oder durch (relationalen) Vertrag begründet werden. Von größerem theoretischem Interesse ist die Frage, ob sich solche Strukturen auch zwischen auf andere Weise unverbundenen Akteuren allein aus wiederholten und auch künftig erwartbaren Interaktionen entwickeln können. Dies wäre nicht gleichzusetzen mit der spieltheoretischen Begründung einer möglichen "Evolution von Kooperation" (Axelrod 1984), welche die unbegrenzte Wiederholung der gleichen (dem Gefangenendilemma entsprechenden) Interaktion voraussetzt. Derart exakte Wiederholungen treten ja in der Realität nur selten auf. Aber etwas Wichtiges wird in der Tat wiederholt, wenn in einer Dauerbeziehung die gleichen Akteure immer wieder miteinander $\mathrm{zu}$ tun haben. In einem früheren Artikel habe ich vorgeschlagen, diesen Aspekt im Konzept eines Zwei-Ebenen-Spiels zu erfassen, in dem zwar auf der ersten Ebene die konkreten Optionen und Payoffs von Mal zu Mal wechseln, während auf der zweiten Ebene ein und dasselbe Spiel unverändert wiederholt wird. Dort geht

10 Die Aussage gilt nicht für den "Network-Exchange"-Ansatz, der auf der Grundlage der "Power-Dependence"-Theorie (Emerson 1962) die Implikationen unterschiedlicher Netzwerk-Strukturen für die Symmetrie oder Asymmetrie von Tauschbeziehungen untersucht (Cook/ Yamagishi 1992). Im gegenwärtigen Kontext müßste man stark asymmetrische Beziehungen als hierarchische Struktur charakterisieren. Darin liegt eine Vereinfachung. Im Rahmen dieses Aufsatzes können jedoch die Koexistenz symmetrischer und asymmetrischer NetzwerkBeziehungen und ihre Implikationen für das Koordinationsproblem nicht weiter untersucht werden. 
es nämlich immer wieder um die gleiche Frage, welcher "Typ von Spieler" der Akteur in dem eben anstehenden Primär-Spiel sein will, und als welchen er seinen Partner einschätzt (Scharpf 1990).

Gemeint sind mit dem spieltheoretischen Begriff des Spielertyps drei Aspekte der Vertrauenswürdigkeit: Wird der Spieler in den dem eigentlichen Spielzug vorausgehenden Verhandlungen lügen oder die Wahrheit sagen; wird er gegebene Versprechen halten oder brechen; und wird er drittens Spielzüge in Betracht ziehen oder ausschließen, die den Partner schädigen müßten? Auch wenn die Bedeutung dieser drei Aspekte der Vertrauenswürdigkeit von Fall zu Fall variieren mag, sind zumindest die ersten beiden konstitutiv für alle Arten sozial produktiver Interaktionen. Ihre Bedeutung ergibt sich aus einem grundlegenden Dilemma der menschlichen Existenz: Einerseits haben Akteure keine direkte Kenntnis der Situationsdeutungen und Absichten ihrer Partner, während die objektive Fähigkeit zur wechselseitigen Schädigung potentiell unbegrenzt erscheint. Wenn aber die Akteure in Reaktion auf die gefährliche Ungewißheit niemandem über den Weg trauen (also nur noch Maximin-Strategien anwenden) würden, so müßten sie andererseits auch auf alle Vorteile der Kooperation und des wechselseitig vorteilhaften Austauschs verzichten (Luhmann 1968; Scharpf 1990). Vertrauen impliziert also nicht nur lebensgefährliche Risiken, sondern auch lebenswichtige Chancen.

Nun besagen spieltheoretische "Folk-Theorems" in der Tat, daß schon die unbefristete Wiederholung von Spielen ausreiche, um Kooperation zu ermöglichen. In den Spielen auf der zweiten Ebene würden demnach rationale Egoisten die Wahrheit sagen und Versprechen halten, weil andernfalls der Partner den Vertrauensbruch bei der nächsten Begegnung bestrafen könnte. Unklar bleibt freilich innerhalb des theoretischen Bezugsrahmens die Rationalität (oder die "subgame-perfectness") der dabei unterstellten Sanktionsbereitschaft der Partner (Güth et al. 1991). Wenn Sanktionen kostspielig sind, dann ist nicht einzusehen, weshalb egoistisch-rationale Akteure (die also nicht von einem emotionalen Bedürfnis nach Rache getrieben werden: Frank 1988) Ressourcen aufwenden oder auf potentielle Vorteile verzichten sollten, nur um alte Rechnungen mit einem früheren Übeltäter zu begleichen.

Plausibel erscheint deshalb die Vertrauensbildung zwischen egoistisch-rationalen Akteuren nur in Situationen, wo eine hochwirksame Sanktion nur geringe Kosten verursacht. Auf Wettbewerbsmärkten und unter Bedingungen hoher Sichtbarkeit können die Käufer mit geringen Kosten einem Verkäufer aus dem Weg gehen, von dem sie einmal betrogen wurden, oder von dem sie wissen, daß er andere betrogen hat (Tullock 1985), und spezialisierte Informationsdienste können auch unter weniger günstigen Umständen hohe 
Sichtbarkeit erzeugen (Milgrom et al. 1990). Wo das zutrifft, haben rationale Akteure guten Grund, ihre Reputation für Vertrauenswürdigkeit nicht zu gefährden, selbst wenn im konkreten Fall Betrug vorteilhafter wäre. Insofern können also stabile Marktbeziehungen durchaus von wechselseitigem Vertrauen geprägt sein, das auch keineswegs "parasitär" von nicht erneuerbaren traditionalen Normbeständen leben muß (so Bell 1976), sondern sich aus den ökonomischen Kalkülen selbst regenerieren kann.

Aber es gibt viele andere Konstellationen, in denen die Akteure einander nicht mit geringen Kosten aus dem Weg gehen können. Das gilt nicht nur für Interaktionen zwischen Ehepartnern und Nachbarn und zwischen Unternehmen bei hoher "Asset-Specificity" (eine Bedingung, auf die sich die Transaktionskosten-Ökonomie konzentriert), sondern es gilt auch zwischen territorial oder funktional spezialisierten und verhältnismäßig immobilen Organisationen wie Gewerkschaften, Interessenverbänden, politischen Parteien und Staaten. In all diesen Konstellationen könnte in der Modell-Welt der Reputationsmechanismus für sich allein nicht wechselseitiges Vertrauen garantieren. Überdies wissen wir ja auch, daß in der realen Welt die Beziehungen zwischen Akteuren, die einander nicht aus dem Weg gehen können, nicht immer durch wechselseitiges Vertrauen, sondern oft auch durch besonderes Mißtrauen geprägt sind.

Erklärungsbedürftig ist also das Nebeneinander von Vertrauen und Mißtrauen in dauerhaften Beziehungen. Was kann das Netzwerk-Konzept dazu beitragen? Die Antwort, die ich anzubieten habe, basiert auf der spekulativen Extrapolation begrenzter empirischer Befunde. In einer Untersuchung der horizontalen Koordination zwischen deutschen Bundesländern (Scharpf/ Benz 1991) fiel uns auf, daß die Akteure generell dazu neigten, ihre besonders bedeutsamen Beziehungen dichotomisch als entweder "kooperativ" oder "kompetitiv" zu definieren. Daraus leite ich die Vermutung ab, daß von den in sozialpsychologischen Experimenten identifizierten möglichen "Interaktionsorientierungen" (MacCrimmon/ Messick 1976; Kelley/ Thibaut 1978; Schulz/ May 1989) in realen Interaktionen nicht alle mit gleicher Häufigkeit auftreten. Insbesondere scheint die (von der ökonomischen Theorie und von der Spieltheorie als Norm unterstellte) "egoistisch-rationale" Orientierung, nach der Akteure sich nur um den eigenen Nutzen kümmern $\left(U=U_{x}\right)$ und gegenüber dem Nutzen ihrer Partner indifferent sind, in bedeutsamen Dauerbeziehungen nicht die Regel zu sein. Bei den kooperativen bzw. kompetitiven Orientierungen dagegen interessieren sich die Akteure durchaus für die Gewinne und Verluste der anderen Spieler - im ersten Fall zählt für sie der gemeinsame Nutzen der Partner $\left(U=U_{x}+U_{\gamma}\right)$, im zweiten der eigene Vorsprung vor dem Konkurrenten $\left(\mathrm{U}=\mathrm{U}_{\mathrm{X}}-\mathrm{U}_{\mathrm{Y}}\right)$. 
Die Neigung, wichtige Beziehungen entweder als kompetitiv oder als kooperativ zu definieren, mag in der evolutionär geprägten menschlichen Natur begründet sein (Messick 1985); sie hat aber auch, wie ich an anderer Stelle gezeigt habe, den Vorteil der radikalen kognitiven Vereinfachung komplexer und ambivalenter Spielsituationen. Mixed-Motive-Konstellationen wie das Prisoner's Dilemma, das Chicken Game oder Battle-of-the-Sexes, die bei egoistisch-rationaler Orientierung schwierige Kalküle erfordern und dennoch keine befriedigende Lösung haben, werden durch die kompetitive Orientierung in einfache Nullsummenspiele, durch die kooperative Orientierung in ebenso einfache Koordinationsspiele transformiert (Scharpf 1988; 1989). Hinzu kommt, so denke ich, ein zweiter Mechanismus, den man als Reaktion begrenzt-rationaler Akteure auf die fundamentale Ungewißheit menschlicher Interaktionen interpretieren kann.

Dauerbeziehungen werden ja fast immer Einzelinteraktionen einschließen, die sich in ihren spieltheoretischen Eigenschaften erheblich voneinander unterscheiden. Einige davon mögen einem Nullsummenspiel entsprechen, einige einem reinen Koordinationsspiel, und viele werden die Struktur von "MixedMotive"-Spielen haben, also etwa dem-Prisoner's Dilemma, dem ChickenGame oder auch dem Assurance-Game entsprechen. Wenn alle diese Spiele jeweils unter vollständiger Information egoistisch-rational gespielt würden, dann würden die Partner manchmal einander unterstützen und manchmal einander schädigen. Bei prinzipiell unvollständiger Information über die Präferenzen und Perzeptionen der anderen Seite freilich wären die Akteure oft nicht in der Lage, die eine Konstellation von der anderen sicher zu unterscheiden. Dann aber brauchte es nur ein geringes Maß an "Risikoaversion" oder eine Tendenz zur Überbewertung möglicher Verluste im Vergleich zu möglichen Gewinnen (Kahneman/ Tversky 1984), um wiederum die extreme Vorsicht als plausibelste Verhaltensregel erscheinen zu lassen.

Weil aber das generalisierte Mißtrauen zu so unattraktiven Ergebnissen führen muß, kann man in der Tat ein universelles menschliches Interesse am Aufbau vertrauensvoller Beziehungen postulieren (Sabel 1992). Angesichts der potentiellen Vielfalt von Spiel-Konstellationen, die von den Akteuren nur unvollständig durchschaut werden können, kann ein solches Interesse seinen Ausdruck freilich nur in der Ausbildung generalisierter Erwartungen finden - und es kann nur verwirklicht werden, wenn diese Erwartungen sich in den nachfolgenden Interaktionen auch regelmäßig bestätigen. Derartige Erwartungen können nicht über alle Beziehungen hinweg einheitlich sein, aber es erscheint ebenso unwahrscheinlich, daß sie vollständig individualisiert werden könnten. Statt dessen scheint es plausibel, sie als empirische Generali- 
sierungen zu interpretieren, die aus den realen Bedingungen besonders häufiger oder besonders wichtiger Interaktionen abgeleitet wurden.

Für kompetitive oder sogar feindselige ${ }^{11}$ Orientierungen erscheint die Vermutung besonders naheliegend, daß sie ihre Grundlage in realen Konstellationen haben, in denen die Partner objektiv inkompatible Ziele verfolgt haben. Wenn solche Erfahrungen mit einer gewissen Häufigkeit auftreten, dann gibt es in der Tat gute Gründe dafür, auch bei allen anderen Begegnungen darauf zu achten, ob der Konkurrent durch das Ergebnis gestärkt oder geschwächt werden könnte (Powell 1991). Die Akteure werden also Vorkehrungen gegen die Möglichkeit treffen, daß der andere sie ungestraft schädigen könnte, und sie werden davon ausgehen, daß auch dieser Gegner auf das gleiche "Sicherheitsdilemma" reagieren muß (Jervis 1978; 1985). Indem sie sich aber gegen "worst-case"-Szenarien zu schützen versuchen, transformieren sie alle einzelnen Interaktionen, unabhängig von deren "objektiv gegebenem" Charakter, in "effektive" Nullsummenspiele. Die Beziehung wird dann insgesamt durch generalisierte Erwartungen der Konkurrenz oder der Feindseligkeit definiert; und diese Erwartungen werden in aller Regel auch durch die nachfolgende Erfahrung bestätigt.

Während aber das generalisierte Mißtrauen sich aus objektiven Interessenkonstellationen gewissermaßen von selbst entwickeln kann, reicht es für die Etablierung generalisierten Vertrauens noch nicht aus, daß Akteure in wichtigen Fällen gemeinsame Interessen verfolgt haben. Schon beim nächsten Spiel könnten ja die Interessen ganz anders liegen. Da die Akteure im Prinzip nicht über wechselseitig zuverlässige Kenntnis ihrer Situationsdeutungen und Absichten verfügen können, verlangt vertrauensvolle Kooperation immer von neuem die Bereitschaft, sich auf verwundbare Positionen einzulassen. Schlimmer noch, bei allfälligen Enttäuschungen könnte die verletzte Partei nicht einmal hinterher zuverlässig wissen, ob es sich um einen kontingenten Interessenkonflikt gehandelt hat (der künftige Kooperation unter günstigeren Umständen nicht hindern würde), oder ob eine böswillige Schädigung vorliegt, die darauf schließen läßt, daß der vermeintliche Partner zum Konkurrenten oder Feind geworden ist (Jervis 1988).

Angesichts des mit der Gewährung von Vertrauen verbundenen hohen Risikos erscheint es deshalb plausibel, daß Akteure auf das unaufhebbare Informationsdefizit zwar nicht mit generalisiertem Mißtrauen reagieren, aber

11 Die feindselige Orientierung unterscheidet sich von der kompetitiven dadurch, daß der Schaden des anderen auch ohne Rücksicht auf den eigenen Vorteil gesucht werden kann; also nicht $\left(U=U_{x}-U_{\gamma}\right)$, sondern $\left(U=-U_{\gamma}\right)$. 
doch mit höheren Anforderungen an die Kriterien der "Vertrauenswürdigkeit" oder, was auf das gleiche hinausläuft, mit einer Absenkung der Schwelle, von der an das Vertrauen entzogen wird. Anstatt sich auf unsichere Erklärungen einzulassen, hätten sie guten Grund, schon auf die tatsächliche Schädigung ihrer Interessen mit Vertrauensentzug zu reagieren. Dementsprechend würden also "kooperative" Beziehungen definiert durch die generalisierte Erwartung, daß die Partner die Schädigung ihrer wechselseitigen Interessenpositionen vermeiden werden. Angesichts der Variabilität ihrer realen SpielKonstellationen, wären solche Erwartungen selbstverständlich völlig unrealistisch, wenn die Partner entsprechend der egoistisch-rationalen Orientierung jeweils nur den eigenen Nutzen maximieren würden. ${ }^{12}$ Generalisiertes Vertrauen erfordert vielmehr die Bereitschaft der Partner, im Interesse der Aufrechterhaltung einer kooperativen Dauerbeziehung im Einzelfall auf eigene Vorteile zu verzichten und sogar Verluste hinzunehmen. Wenn diese Definition die wechselseitigen Erwartungen bestimmt, dann mögen unbeabsichtigte Fehler nach ausreichender Klärung immer noch verziehen werden, aber alle anderen Enttäuschungen würden in aller Regel als Aufkündigung der kooperativen Beziehung selbst interpretiert, die auf der anderen Seite auch den allgemeinen Vertrauensentzug rechtfertigt.

Generalisiertes Vertrauen kann also leicht zerstört werden. Wo es aber existiert, bringt es den Beteiligten außerordentliche Vorteile. Es erlaubt ihnen, verwundbare Positionen einzunehmen und sich auch unter Bedingungen der unvollkommenen Information auf hochriskante (aber potentiell auch hochprofitable) "Mixed-Motive"-Transaktionen einzulassen. Aber trotz dieser außerordentlichen Vorteile erscheint es unwahrscheinlich, daß in der Mehrzahl der Dauerbeziehungen generalisiertes Vertrauen herrschen könnte. Angesichts der unaufhebbaren Ungewißheit bleibt Vertrauen eine riskante Entscheidung, die man nicht leichthin treffen wird (Sabel 1993). Noch wichtiger erscheint die Tatsache, daß eigene Vertrauenswürdigkeit nur durch aufwendige und leichtverderbliche Investitionen erworben werden kann, deren Kosten überdies steil ansteigen müßten, wenn ein Akteur sich mit konfligierenden Erwartungen von zwei oder mehr Vertrauensgebern zur gleichen Zeit konfrontiert sieht. Der Versuch, "everybody's darling" sein zu wollen, würde in aller Regel durch die hohen Opportunitätskosten kooperativer Beziehungen vereitelt.

12 In der sozialpsychologischen Literatur wird zwischen einer "kooperativen" (U $\left.=U_{X}+U_{Y}\right)$ und einer "altruistischen" Interaktionsorientierung $\left(U=U_{Y}\right)$ unterschieden. Die hier abgeleitete Definition ist weniger anspruchsvoll. Sie erlaubt die Verfolgung eigener Interessen unter der Voraussetzung, daß der Partner dadurch nicht geschädigt wird. 
Aus diesem Grund bleibt durchaus Raum für eine dritte, "indifferente" Kategorie von Beziehungen, in denen die Akteure sich wechselseitig weder als Freunde noch als Feinde definieren. Während sie einander große Opfer nicht zumuten können, wären hier kleinere reziproke Gefälligkeiten durchaus erwartbar. Deshalb können die Partner indifferenter Dauerbeziehungen auch erwarten, daß der andere die eigenen Interessen nicht gedankenlos und unnötigerweise verletzen werde (Colman 1982: 38); und sie werden vermutlich auf eine "kompetitive" Orientierung umschalten, wenn selbst diese Minimalerwartungen verletzt werden. In andern Worten: Die Interaktionsorientierungen in indifferenten Dauerbeziehungen werden in etwa den Standardannahmen eines eigeninteressierten, aber nicht-opportunistischen Rationalverhaltens entsprechen, wie es in der ökonomischen Theorie gemeinhin unterstellt wird.

Das resultierende Beziehungsmuster wird also vermutlich eine stark selektive Struktur haben. Angesichts ihrer hohen Kosten werden kooperative Bindungen nicht leichthin eingegangen, und das gleiche wird auch für kompetitive Beziehungen gelten, deren Unterhaltung ebenfalls dauernde Aufmerksamkeit und Anstrengung erfordert. Dementsprechend werden sowohl kooperative als auch kompetitive Orientierungen sich vor allem in Beziehungen von hoher intrinsischer Bedeutung, mit potentiell hohen Vorteilen oder hoher Verwundbarkeit herausbilden - also in Beziehungen, für welche die Unterstellung "Wer nicht für mich ist, muß wider mich sein" eine erhebliche Plausibilität besitzt. Rationale Indifferenz dagegen wird eher in Beziehungen von geringerer intrinsischer Bedeutung auftreten. Darüberhinaus besagen psychologische Theorien des "strukturellen Gleichgewichts", daß sich in Populationen von interdependenten Akteuren Cliquen herausbilden werden, deren interne Beziehungen positiv (vertrauensvoll) sind, während zwischen diesen Cliquen negative oder indifferente Beziehungen vorherrschen. ${ }^{13}$ In der experimentellen Prüfung haben diese analytisch gewonnenen Hypothesen nicht besonders gut abgeschnitten (Hummell/ Soudeur 1987) und auch historische Studien haben in Systemen internationaler Allianzen keine dauerhafte Tendenz zum "strukturellen Gleichgewicht" entdecken können (McDonald/ Rosecrance

13 Die Heidersche Gleichgewichtstheorie läßt nur positive oder negative Beziehungen zu. Sie unterstellt eine psychologische Tendenz zur Stress-Vermeidung, nach welcher der Freund eines Freundes als Freund, der Feind eines Freundes als Feind, der Freund eines Feindes als Feind und der Feind eines Feindes als Freund definiert werden. Im Ergebnis würde durch diese Regeln die Population in zwei feindliche Lager geteilt (Heider 1946; Cartwright/ Harary 1956). Wenn auch indifferente Beziehungen zugelassen werden, dann teilt sich die Population in mehrere Cliquen von Freunden, die von anderen durch negative oder indifferente Beziehungen getrennt sind (Hummell/ Soudeur 1987). 
1985). ${ }^{14}$ Generell können wir deshalb nur davon ausgehen, daß kooperative Beziehungen höchst selektive Netzwerk-Strukturen bilden werden, die jedoch nicht isoliert existieren. Die Mitglieder solcher Netzwerke werden auch mit anderen Akteuren interagieren, mit denen sie durch indifferente oder kompetitive Beziehungen verbunden sind.

\section{Positive und negative Koordination in Netzwerken}

Damit kehre ich zu der Ausgangsfrage zurück: Was kann die Existenz kooperativer Netzwerke zur Effektivität der nicht-hierarchischen Koordination in der modernen Gesellschaft beitragen? Für die positive Koordination liegt die Antwort auf der Hand: sie wird wesentlich erleichtert, wenn Verhandlungen zwischen Parteien geführt werden können, deren Dauerbeziehung durch kooperative Orientierungen definiert wird. Generalisiertes Vertrauen und ein generalisierter Willen zur Vertrauenswürdigkeit schließen opportunistische Verhandlungsstrategien aus, die den Kern des "Verhandlungsdilemmas" ausmachen; und wenn Verteilungskonflikte nicht ohnehin durch die Erwartung von "diffuser Reziprozität" (Keohane 1986) verdrängt werden, werden sie doch zumindest abgemildert durch das weithin vorauszusetzende Einvernehmen über die anwendbaren Normen der Verteilungsgerechtigkeit. Klar ist freilich auch, daß diese wohltätigen Wirkungen nur dann eintreten können, wenn der aktuelle Koordinationsbedarf übereinstimmt mit der gegebenen Struktur eines kooperativen Netzwerks.

In anderen Worten, die "näherungsweise Zerlegbarkeit" oder "Modularität" ist nicht nur für hierarchische Organisation ein Grundproblem, sondern auch für Netzwerkstrukturen. Zwar ist in Netzwerken, deren Struktur von der perzipierten Intensität bilateraler Interdependenzen geprägt wird, die Kongruenz zwischen Struktur und tatsächlichem Koordinationsbedarf vermutlich größer als in formalen Organisationen. Aber angesichts der zunehmenden Veränderlichkeit realer Interdependenz-Beziehungen kann dies keineswegs die vollkommene Übereinstimmung garantieren. Auch in Netzwerkstrukturen gibt es erhebliche Veränderungswiderstände, und Freunde könnten nicht Freunde sein, wenn Freundschaftsbeziehungen nach dem jeweils neuesten Stand des perzipierten Koordinationsbedarf wechseln müßten.

14 Die Untersuchungen zur Struktur europäischer Allianzen im letzten Drittel des neunzehnten Jahrhunderts zeigen aber doch eine sozusagen "natürliche" Tendenz zur Polarisierung, das der Heiderschen Theorie entspricht. Diese Tendenz wurde nur zeitweilig überdeckt durch Bismarcks Strategie der sich überkreuzenden Allianzen, die von seinen Nachfolgern nicht mehr durchgehalten werden konnte. 
Deshalb ist es wahrscheinlich, daß immer wenigstens einige der Akteure, auf deren Zustimmung man für erfolgreiche positive Koordination angewiesen wäre, nicht zu den Mitgliedern eines bereits existierenden kooperativen Netzwerks gehören werden. Dies ist gewiß noch nicht das Todesurteil für die Chancen der positiven Koordination. Aber die Beteiligung von "Außenseitern" wird nun wieder die Transaktionskosten von Verhandlungen erhöhen und die Einigung erschweren - und sie wird zugleich, als Folge des Problems der großen Zahl, die Ausdehnung der Gruppe begrenzen, innerhalb der erfolgreiche positive Koordination durch Verhandlungen erreicht werden kann.

Im Gegensatz dazu wird die negative Koordination gerade dann an Bedeutung gewinnen, wenn die Struktur kooperativer Netzwerke und das Muster aktueller Probleminterdependenzen sich überkreuzen. Die Rechtsordnung bietet ja außerhalb der Grenzen formaler Organisationen nur einen sehr begrenzten Schutz gegen die externen Effekte einseitigen Handelns. Das Strafrecht und das Privatrecht der unerlaubten Handlung bieten nur Abhilfe gegen Eingriffe in Leben, körperliche Unversehrtheit und Freiheit und in gewisse wohldefinierte eigentumsähnliche Rechte. Darüberhinaus gibt es keine allgemeine Rechtspflicht, sich um die Interessen potentiell betroffener Dritter zu kümmern. Private Organisationen sind rechtlich nicht gehindert, sich wechselseitig Kunden, Klienten, Mitglieder oder Investoren abzuwerben; und öffentliche Organisationen können ihre eigene Politik ohne Rücksicht auf deren externe Effekte für andere Zuständigkeitsbereiche verfolgen. Erst recht trifft dies auf der internationalen Ebene zu. Und selbst wenn die einzelnen Akteure gerne Rücksicht üben würden, wird der kollektive Egoismus sich in aller Regel dann durchsetzen, wenn Entscheidungen im Rahmen multilateraler Verhandlungen getroffen werden müssen. Da Einvernehmen unter den unmittelbar beteiligten Verhandlungspartnern schon schwer genug zu erreichen ist, wird die "unnötige" Rücksicht auf die Interessen von Außenseitern kaum eine Chance erhalten.

So gesehen ist es ein Glück, daß der Kreis der Verhandlungsteilnehmer üblicherweise nicht mit den Grenzen existierender kooperativer Netzwerke übereinstimmt und daß deshalb viele Verhandlungspartner auch kooperative Beziehungen zu Dritten unterhalten, die an den Verhandlungen nicht unmittelbar beteiligt sind. Wo immer das der Fall ist, wird der mit dem Versuch der positiven Koordination implizierte kollektive Egoismus konterkariert durch das individuelle Eigeninteresse von Verhandlungspartnern, die ihre Vertrauensbeziehung zu außenstehenden Dritten schützen müssen. Sie müssen, während sie das bestmögliche Ergebnis in den aktuellen Verhandlungen zu erreichen suchen, zugleich immer die Auswirkungen einer möglichen 
Einigung auf die Interessenposition ihrer externen Partner im Blick haben. Wenn diese verletzt werden sollte, so könnte dies Vertrauensbeziehungen gefährden, die durch kostspielige Investitionen in der Vergangenheit aufgebaut wurden und von denen noch ein dauerhafter Strom künftigen Nutzens erwartet werden kann. ${ }^{15}$ Es wird vermutlich nicht viele Fälle geben, in denen der in aktuellen Verhandlungen erreichbare Vorteil einen so hohen Preis wert wäre.

Mit anderen Worten, negative Koordination funktioniert in Netzwerkstrukturen mehr oder minder ebenso wie in hierarchischen Strukturen. Sie sichert die Vermeidung (mancher) negativer Externalitäten und sie begrenzt zugleich den Handlungsraum, und damit die erreichbaren Wohlfahrtsgewinne der durch Verhandlungen angestrebten positiven Koordination. Trotzdem sollte man die Opportunitätskosten der Negativkoordination nicht überschätzen. Sie werden einmal begrenzt durch die Tatsache, daß kooperative Netzwerke nicht hierarchisch oktroyiert werden, sondern durch Selbstorganisation entstehen. Da die Beziehungen von den unmittelbar beteiligten Akteuren selbst geschaffen werden, werden sie in der Regel die von den Akteuren perzipierten wichtigsten strukturellen Interdependenzen repräsentieren; und da ihre Veränderung nicht von formellen Vereinbarungen abhängt, wird ihre Struktur sich der Entwicklung tatsächlicher Interdependenzen im Laufe der Zeit anpassen. Überdies ist ja auch der Kreis der Verhandlungsteilnehmer, unter denen positive Koordination angestrebt wird, keineswegs unveränderlich. Seine Zusammensetzung wird sich von vornherein an den perzipierten Interdependenzen orientieren, aber er kann notfalls auch ad hoc erweitert oder verändert werden.

15 Ähnliche Restriktionen können auch aus der Existenz kompetitiver Beziehungen resultieren, die einige Verhandlungspartner veranlassen mag, Lösungen zu vermeiden, die den Konkurrenten stärken könnten. Das ist offenbar gemeint, wenn man sagt, daß die Bundesrepublik immer als "stiller Verhandlungspartner" dabei war, wenn die DDR internationale Vereinbarungen anstrebte. 


\section{Zusammenfassung}

Die Existenz kooperativer Netzwerk-Strukturen ermöglicht Formen der positiven und negativen Selbstkoordination, die durchaus vergleichbar sind mit der Selbstkoordination in hierarchischen Organisationen. In beiden Fällen reduziert die Einbettung von Verhandlungen in einen vorhandenen strukturellen Rahmen die Transaktionskosten und erhöht damit die Reichweite und die Wirksamkeit von positiver Koordination. Noch wichtiger scheint es, daß die Einbettung negative Koordination ermöglicht und so auch die Interessen von Akteuren schützt, die nicht unmittelbar an aktuellen Verhandlungen beteiligt sind. In der Kombination erhöhen die erörterten Mechanismen deshalb die potentielle Reichweite koordinierten Verhaltens weit über die Grenzen hinaus, innerhalb der hierarchische Koordination oder Verhandlungskoordination für sich allein Erfolg haben könnten.

Freilich bedeutet die strukturelle Einbettung auch, daß die Ergebnisse der Koordination von der jeweiligen strukturellen Konfiguration bestimmt werden. Selbstkoordination durch Verhandlungen im Schatten der Hierarchie ist abhängig von der jeweils gegebenen Struktur von Autoritätsbeziehungen (oder asymmetrischen Machtbeziehungen) und von den Grenzen formaler Organisationen. Und auch wo Netzwerkstrukturen über Organisationsgrenzen hinausreichen, ist ihre Wirksamkeit mindestens ebenso selektiv. Sie hängt ab von der jeweils gegebenen Verteilung starker und schwacher Dauerbeziehungen zwischen formell unabhängigen individuellen und korporativen Akteuren. Auch wenn das Gesamtniveau der Koordiniertheit wesentlich erhöht wird, gibt es also keinen Grund für die Annahme, daß alle oder auch nur die meisten Chancen zur Optimierung auch tatsächlich genutzt werden oder daß alle oder die meisten Interessen gegen die negativen Externalitäten von Entscheidungen an anderer Stelle geschützt werden. Mit anderen Worten, auch das Konzept der eingebetteten Verhandlungen verspricht nicht die Verwirklichung eines Wohlfahrtsoptimums unter realen Bedingungen. Aber es verspricht doch eine bessere Erklärung für den überraschend hohen Grad an tatsächlich wirksamer Handlungskoordination und Erwartungssicherheit jenseits der engen Grenzen, in denen Markt und Hierarchie allein die Turbulenz interdependenter Interaktionen bewältigen könnten. 


\section{Literatur}

Aivazian, Varouj A./ Jeffrey L. Callen, 1981: The Coase Theorem and the Empty Core. In: Journal of Law and Economics 24, 175-182.

Aivazian, Varouj A./ Jeffry L. Callen/ Irwin Lipnowski, 1987: The Coase Theorem and Coalitional Stability. In: Econometrica 54, 517-520.

Alchian, Armen A./ Harold Demsetz, 1972: Production, Information Costs, and Economic Organization. In: American Economic Review 62, 777-795.

Axelrod, Robert, 1984: The Evolution of Cooperation. New York: Basic Books.

Baldwin, David A., 1990: Politics, Exchange, and Cooperation. In: Bernd Marin (ed.), Generalized Political Exchange. Antagonistic Cooperation and Integrated Policy Circuits. Frankfurt a.M.: Campus, 101-118.

Bell, Daniel, 1976: The Cultural Contradictions of Capitalism. London: Heinmann.

Bianco, William T./ Robert H. Bates, 1990: Cooperation by Design: Leadership, Structure, and Collective Dilemmas. In: American Political Science Review 84, 133-147.

Brennan, Geoffrey/ James M. Buchanan, 1985: The Reason of Rules. Constitutional Political Economy. Cambridge: Cambridge University Press.

Cartwright, Dorwin/ Frank Harary, 1956: Structural Balance: A Generalization of Heider's Theory. In: Psychology Review 63, 277-293.

Chisholm, Donald, 1989: Coordination Without Hierarchy. Informal Structures in Multiorganizational Systems. Berkeley: University of California Press.

Coase, Ronald H., 1960: The Problem of Social Cost. In: Journal of Law and Economics 3, 1-44.

Coase, Ronald H., 1981: The Coase Theorem and the Empty Core: A Comment. In: Journal of Law and Economics 24, 183-187.

Colman, Andrew M., 1982: Game Theory and Experimental Games. The Study of Strategic Interaction. Oxford: Pergamon Press.

Cook, Karen S./ Toshio Yamagishi, 1992: Power in Exchange Networks: A Power-Dependence Formulation. In: Social Networks 14, 245-267.

Czada, Roland, 1991: Regierung und Verwaltung als Organisatoren gesellschaftlicher Interessen. In: Hans-Hermann Hartwich/ Göttrik Wewer (Hrsg.): Regieren in der Bundesrepublik III. Systemsteuerung und "Staatskunst". Opladen: Leske u. Budrich, 151-173. 
Dearborn, DeWitt C./ Herbert A. Simon, 1958: Selective Perception: A Note on the Departmental Identification of Executives. In: Sociometry 21, 140-144.

Dinar, Ariel/ Aharon Ratner/ Dan Yaron, 1992: Evaluating Cooperative Game Theory in Water Resources. In: Theory and Decision 32, 1-20.

Dryzek, John R., 1992: How Far is it from Virginia and Rochester to Frankfurt? Public Choice as Critical Theory. In: British Journal of Political Science 22, 397-418.

Emerson, Richard M., 1962: Power-Dependence Relations. In: American Sociological Review 27, 31-41.

Frank, Robert H., 1988: Passion Within Reason. The Strategic Role of Emotions. New York: W.W. Norton.

Gastel, M.A.J.J. van/ J.H.P. Paelinck, 1992: Generalization of Solution Concepts in Conflict and Negotiation Analysis. In: Theory and Decision $32,65-77$.

Groom, A.J.R., 1991: No Compromise: Problem-Solving in a Theoretical Perspective. In: International Social Science Journal 127, 77-86.

Güth, Werner/Wolfgang Leininger/ Gunter Stephan, 1991: On Supergames and Folk Theorems: A Conceptual Discussion. In: Reinhard Selten (ed.), Game Equilibrium Models II. Methods, Morals, and Markets. Berlin: Springer, 56-70.

Haas, Ernst B., 1980: Why Collaborate? Issue Linkage and International Regimes. In: World Politics 32, 357-405.

Häusler, Jürgen/ Hans-Willy Hohn/ Susanne Lütz, 1993: The Architecture of an R \& D Collaboration. In: Fritz W. Scharpf (ed.), Games in Hierarchies and Networks. Analytical and Empirical Approaches to the Study of Governance Institutions. Frankfurt a.M.: Campus, 211-249.

Hardin, Russell, 1988: Morality within the Limits of Reason. Chicago: University of Chicago Press.

Harsanyi, John C., 1977: Rational Behavior and Bargaining Equilibrium in Games and Social Situations. Cambridge: Cambridge University Press.

Hausman, Daniel M., 1991: Is Utilitarianism Useless? In: Theory and Decision $30,273-278$.

Hayek, Friedrich A. von, 1945: The Use of Knowledge in Society. In: American Economic Review 35, 519-530.

Heider, Fritz, 1946: Attitudes and Cognitive Organization. In: Journal of Psychology 21, 197-112. 
Hummell, Hans J./ Wolfgang Soudeur, 1987: Triaden- und Triplettzensus als Mittel der Strukturbeschreibung. In: Franz Urban Pappi (Hrsg.), Methoden der Netzwerkanalyse. München: Oldenburg, 129-161.

Jervis, Robert, 1978: Cooperation Under the Security Dilemma. In: World Politics 30, 167-214.

Jervis, Robert, 1985: From Balance to Concert: A Study of International Security Cooperation. In: World Politics 38, 58-79.

Jervis, Robert, 1988: Realism, Game Theory, and Cooperation. In: World Politics $40,317-349$.

Kahneman, Daniel/ Amos Tversky, 1984: Choices, Values, and Frames. In: American Psychologist 39, 341-350.

Kalai, Ehud/ Meir Smorodinsky, 1975: Other Solutions to Nash's Bargaining Problem. In: Econometrica 43, 513-518.

Kaldor, Nicholas, 1939: Welfare Propositions of Economics and Interpersonal Comparisons of Utility. In: The Economic Journal 49, 549-552.

Kelley, Harold H./ John W. Thibaut, 1978: Interpersonal Relations. A Theory of Interdependence. New York: John Wiley.

Keohane, Robert O., 1986: Reciprocity in International Relations. In: International Organization 40, 1-27.

Lax, David A./ James K. Sebenius, 1986: The Manager as Negotiator. Bargaining for Cooperation and Competitive Gain. New York: Free Press.

Lindberg, Leon N./ John L. Campbell/ J. Rogers Hollingsworth, 1991: Economic Governance and the Analysis of Structural Change in the American Economy. In: John L. Campbell/ J. Rogers Hollingsworth/ Leon N. Lindberg (eds.), Governance of the American Economy. Cambridge: Cambridge University Press, 3-34.

Lindberg, Leon N./ John L. Campbell, 1991: The State and the Organization of Economic Activity. In: John L. Campbell/ J. Rogers Hollingsworth/ Leon N. Lindberg (eds.), Governance of the American Economy. Cambridge: Cambridge University Press, 356-395.

Luhmann, Niklas, 1968: Vertrauen. Ein Mechanismus der Reduktion sozialer Komplexität. Stuttgart: Ferdinand Enke.

MacCrimmon, Kenneth R./ David M. Messick, 1976: A Framework for Social Motives. In: Behavioral Science 21, 86-100.

MacGinnis, Michael D., 1986: Issue Linkage and the Evolution of International Cooperation. In: Journal of Conflict Resolution 30, 141-170. 
Macneil, Ian R., 1978: Contracts: Adjustment of Long-term Economic Relations Under Classical, Neoclassical, and Relational Contract Law. In: Northwestern University Law Review 72, 854-905.

March, James G./ Johan P. Olsen, 1989: Rediscovering Institutions. The Organizational Basis of Politics. New York: Free Press.

Mayntz, Renate/ Fritz W. Scharpf, 1975: Policy-Making in the German Federal Bureaucracy. Amsterdam: Elsevier.

McDonald, H. Brooke/ Richard Rosecrance, 1985: Alliance and Structural Balance in the International System. In: Journal of Conflict Resolution 29, 57-82.

Messick, David M., 1985: Social Interdependence and Decision Making: In: George Wright (ed.), Behavioral Decision Making. New York: Plenum Press, $87-109.2$

Milgrom, Paul/ John Roberts, 1990: Bargaining Costs, Influence Costs, and the Organization of Economic Activity. In: James E. Alt/ Kenneth A. Shepsle (eds.), Perspectives on Positive Political Economy. Cambridge: Cambridge University Press, 57-89.

Milgrom, Paul/ Douglass C. North/ Barry R. Weingast, 1990: The Role of Institutions in the Revival of Trade: The Law Merchant, Private Judges, and the Champagne Fairs. In: Economics and Politics 2, 1-23.

Miller, Gary J., 1992: Managerial Dilemma. The Political Economy of Hierarchy. Cambridge: Cambridge University Press.

Mirbach, Thomas, 1992: Eine kybernetische Auflösung des Repräsentationsproblems? In: Zeitschrift für Parlamentsfragen 23, 658-672.

Nash, John F., Jr., 1950: The Bargaining Problem. In: Econometrica 18, 155162.

Osborne, Martin J./ Ariel Rubinstein, 1990: Bargaining and Markets. San Diego: Academic Press.

Ouchi, William G., 1984: The M-Form Society. How American Teamwork Can Recapture the Competitive Edge. Reading: Addison-Wesley.

Powell, Robert, 1991: Absolute and Relative Gains in International Relations Theory. In: American Political Science Review 85, 1303-1320.

Pruitt, Dean G., 1981: Negotiation Behavior. New York: Academic Press.

Riker, William H., 1982: Liberalism Against Populism. A Confrontation Between the Theory of Democracy and the Theory of Social Choice. San Francisco: W.H. Freeman. 
Sabel, Charles F., 1992: Studied Trust: Building New Forms of Co-Operation in a Volatile Economy. In: Frank Pyke/ Werner Sengenberger (eds.), Industrial Districts and Local Economic Regeneration. Geneva: International Institute for Labour Studies, 215-250.

Sabel, Charles F., 1993: Constitutional Ordering in Historical Context. In: Fritz W. Scharpf (ed.), Games in Hierarchies and Networks. Analytical and Empirical Approaches to the Study of Governance Institutions. Frankfurt a.M.: Campus, 65-123.

Scharpf, Fritz W., 1972: Komplexität als Schranke der politischen Planung. In: Politische Vierteljahresschrift, Gesellschaftlicher Wandel und politische Innovation, Sonderheft 4, 168-192.

Scharpf, Fritz W., 1977: Does Organization Matter? Task Structure and Interaction in the Ministerial Bureaucracy. In: Organization and Administrative Sciences 8, 149-168.

Scharpf, Fritz W., 1988: Verhandlungssysteme, Verteilungskonflikte und Pathologien der politischen Steuerung. In: Manfred G. Schmidt (Hrsg.), Staatstätigkeit. International und historisch vergleichende Analysen. Politische Vierteljahresschrift, Sonderheft 19, 61-87.

Scharpf, Fritz W., 1989: Decision Rules, Decision Styles, and Policy Choices. In: Journal of Theoretical Politics 1, 149-176.

Scharpf, Fritz W., 1990: Games Real Actors Could Play: The Problem of Mutual Predictability. In: Rationality and Society 2, 471-494.

Scharpf, Fritz W., 1991: Die Handlungsfähigkeit des Staates am Ende des zwanzigsten Jahrhundert. In: Politische Vierteljahresschrift 32, 621-634.

Scharpf, Fritz W., 1992: Koordination durch Verhandlungssysteme: Analytische Konzepte und institutionelle Lösungen. In: Arthur Benz/ Fritz W. Scharpf/ Reinhard Zintl, Horizontale Politikverflechtung. Zur Theorie von Verhandlungssystemen. Frankfurt a.M.: Campus, 51-96.

Scharpf, Fritz W./ Arthur Benz, 1991: Kooperation als Alternative zur Neugliederung? Zusammenarbeit zwischen den norddeutschen Ländern. Baden-Baden: Nomos.

Schuessler, Rudolf, 1988: Der homo oeconomicus als skeptische Fiktion. In: Kölner Zeitschrift für Soziologie und Sozialpsychologie 40, 447-463.

Schulz, Ulrich/ Theo May, 1989: The Recording of Social Orientations with Ranking and Pair Comparison Procedures. In: European Journal of Social Psychology 19, 41-59.

Sebenius, James K., 1983: Negotiating Arithmetic: Adding and Subtracting Issues and Parties. In: International Organization 37, 281-316. 
Selten, Reinhard, 1986: Institutional Utilitarianism. In: Franz-Xaver Kaufmann/ Giandomenico Majone/ Vincent Ostrom (eds.), Guidance, Control, and Evaluation. The Bielfeld Interdisciplinary Project. Berlin: de Gruyter, 251263.

Simon, Herbert A., 1951/ 1957: A Formal Theory of the Employment Relation. In: Herbert A. Simon, Models of Man. Social and Rational. New. York: John Wiley, 183-195.

Simon, Herbert A., 1962: The Architecture of Complexity. In: Proceedings of the American Philosophical Society 106, 467-482.

Simon, Herbert A., 1964: On the Concept of Organizational Goal. In: Administrative Science Quarterly 9, 1-22.

Simon, Herbert A., 1973: The Organization of Complex Systems. In: Howard H. Pattee (ed.), Hierarchy Theory. The Challenge of Complex Systems. New York: Braziller, 1-27.

Simon, Herbert A., 1991: Organizations and Markets. In: Journal of Economic Perspectives 5, 25-44.

Stein, Arthur, 1980: The Politics of Linkage. In: World Politics 32, 62-81.

Streeck, Wolfgang/ Philippe C. Schmitter, 1985: Community Market, State and Associations? In: European Sociological Review 1, 119-138.

Thompson, James D., 1967: Organizations in Action. Social-Science Bases of Administrative Theory. New York: McGraw-Hill.

Tullock, Gordon, 1985: Adam Smith and the Prisoners' Dilemma. In: Quarterly Journal of Economics C, Supplement, 1073-1081.

Weingast, Barry, 1989: The Political Institutions of Representative Government: Legislatures. In: Zeitschrift für die gesamte Staatswissenschaft 145, 693703.

Williamson, Oliver E., 1985: The Economic Institutions of Capitalism. New York: Free Press.

Williamson, Oliver E./ William G. Ouchi, 1981: The Markets and Hierarchies and Visible Hand Perspectives. In: Andrew H. Van de Ven/William F. Joyce (eds.), Perspectives on Organization Design and Behavior. New York: John Wiley, 347-370.

Zintl, Reinhard, 1992: Kooperation und Aufteilung des Kooperationsgewinns bei horizontaler Politikverflechtung. In: Arthur Benz/ Fritz W. Scharpf/ Reinhard Zintl, Horizontale Politikverflechtung. Zur Theorie von Verhandlungssystemen. Frankfurt a.M.: Campus, 97-146. 